\title{
Guaranteeing Synchronous Message Deadlines with the Timed Token Medium Access Control Protocol
}

$-$
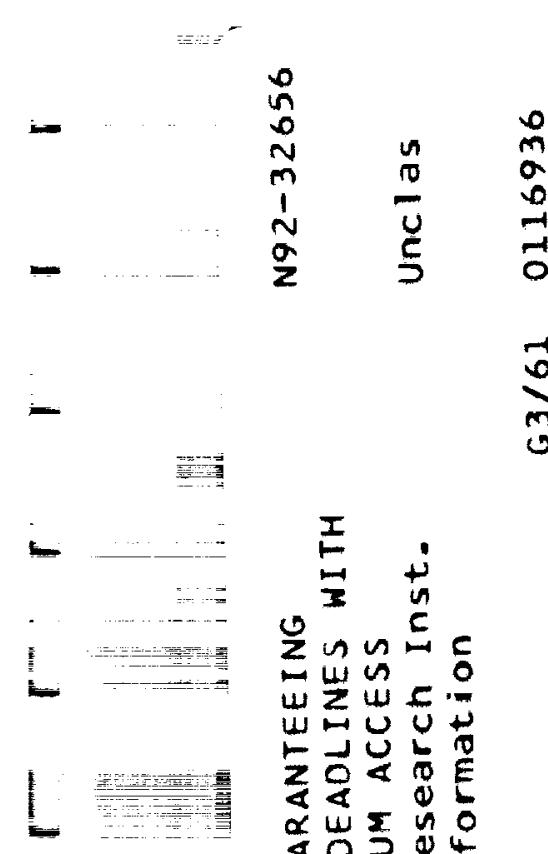

$\sum_{\ln }^{I}$

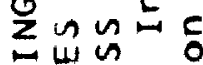

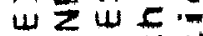

山四U⿺

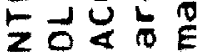

< $\frac{1}{4} 5$

$\alpha w \Sigma \omega_{4}$

$\$ 0 \geqq \alpha$

山员可

0

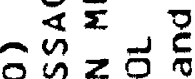

-

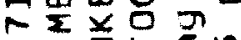

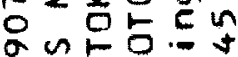

の

$\rightarrow 30$

o 24 은

Uह

$\leq \frac{\alpha}{I}=\alpha \dot{0}$

《I

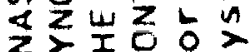

ZथाU⿺乚一

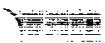

0
0
0
0
0
0

$\underbrace{n}_{0}$

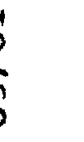

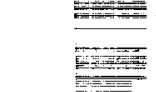

Е
Voldosid

$1 N-6 /-C / 2$

116936

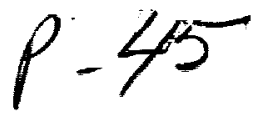

Gopal Agrawal

Baio Chen

Wei Zhao

Texas A\&M University

Sadegh Davari

University of Houston-Clear Lake

March 22, 1992

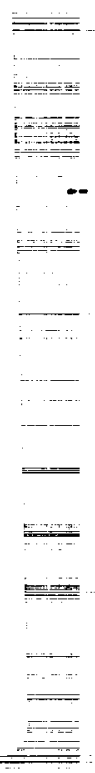

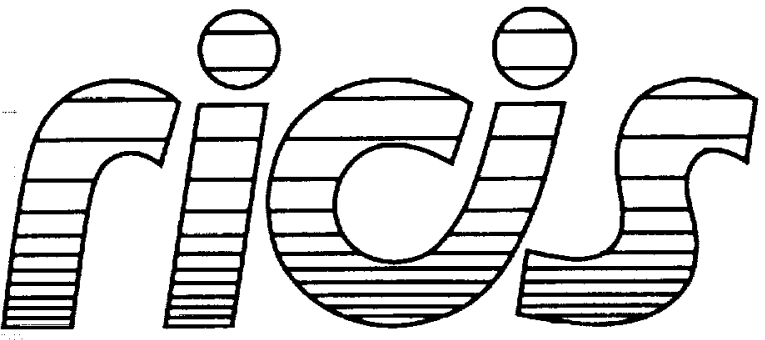

Research institute for Computing and Information Systems University of Houston-Clear Lake 


\section{The RICIS Concept}

The University of Houston-Clear Lake established the Research Institute for Computing and Information Systems (RICIS) in 1986 to encourage the NASA Johnson Space Center (JSC) and local industry to actively support research in the computing and information sciences. As part of this endeavor, UHCL proposed a partnership with JSC to jointly define and manage an integrated program of research in advanced data processing technology needed for JSC's main missions, including administrative, engineering and science responsibilities. JSC agreed and entered into a continuing cooperative agreement with UHCL beginning in May 1986, to jointly plan and execute such research through RICIS. Additionally, under Cooperative Agreement NCC 9-16, computing and educational facilities are shared by the two institutions to conduct the research.

The UHCL/RICIS mission is to conduct, coordinate, and disseminate research and professional level education in computing and information systems to serve the needs of the government, industry. community and academia. RICIS combines resources of UHCL and its gateway afnliates to research and develop materials, prototypes and publications on topics of mutual interest to its sponsors and researchers. Within UHCL, the mission is being implemented through interdisciplinary involvement of faculty and students from each of the four schools: Business and Public Administration, Education, Human Sciences and Humanitjes, and Natural and Applied Sciences. RICIS also collaborates with industry in a companion program. This program is focused on serving the research and advanced development needs of Industry.

Moreover, UHCL established relationships with other universities and research organizations, having common research interests, to provide additional sources of expertise to conduct needed research. For example, UHCL has entered into a special partnership with Texas A\&M University to help oversee RICIS research ant education programs, while other research organizatlons are involved via the "gateway" concept.

A major role of RICIS then is to find the best match of sponsors, researchers and research objectives to advance knowledge in the computing and information sciences. RICIS, working Jointly with its sponsors, advises on research needs, recommends principals for conducting the research, provides technical and administrative support to coordinate the research and integrates technical results into the goals of UHCL, NASA/JSC and industry. 
Guaranteeing Synchronous Message Deadlines with the Timed Token Medium
Access Control Protocol 
$$
=
$$$$
E
$$$$
\equiv
$$ 


\section{RICIS Preface}

This research was conducted by Gopal Agrawal, Baio Chen and Wei Zhao of Texas A\&M University and Dr. Sadegh Davari of the Department of Computer Science at the University of Houston-Clear Lake. The research was supported by the RICIS Program Office and in part by an Engineering Excellence grant from Texas A\&M University.

RICIS research support funds are derived from Cooperative Agreement NCC 9-16 between the NASA Johnson Space Center and the University of Houston-Clear Lake.

The views and conclusions contained in this report are those of the authors and should not be interpreted as representative of the official policies, either express or implied, of UHCL, RICIS, Texas A\&M, NASA or the United States Government. 
$$
=
$$$$
\overline{\bar{E}}
$$ 


\title{
Guaranteeing Synchronous Message Deadlines with the Timed Token Medium Access Control Protocol*
}

\author{
Cropal Agrawal. Baio Chen. and Wei Zhao \\ Department of Computer Science \\ Texas A. M T niversity \\ College Station. TX T-S $\$ 3$ \\ Sadegh Darari \\ Department of Computer Science \\ University of Houston - Clear Lake \\ Houstonl. T. T70.58
}

March 22. 1992

\begin{abstract}
We study the prohlem of guaranteeing synchronous message deadlines in token ring networks where the timed token medium access control protocol is employed. Synchronous capacity defined as the maximum time for which a node can transmit its synchronous messages every time it receives the token. is a key parameter in the control of sunchronous message transmission. To ensure the transmission of sychronons messages before their deadlines stnchronous capacities must be proprerly allocated to individual nodes. We address the issue of appropriate allocation of the srnchronous capacities. Sereral synchronous capacity allocation schemes are analyzed in terms of their ability to satisfy deadline constraints of syuchronous messages. We show that an inappropriate allocation of the synchronous capacities could cause message deadlines to be missed. even if the sruchronous traffic is extremely low. We propose a scheme. called the normalist proportional allocation sheme. which can guarantee the synchronous message deadlines for sruchronous traffic of up to $33 \%$ of arailahle utilization. To date. no ot her suchronous capacity allocation scheme has been reported to achieve such substantial performance.

Another major contribution of this paper is an extension to the previous work on the hounded token rotation time. We prove that the time elapod between aly $r$ conserutive visits to a particular node is hounded by r. TTRT where TTRT is the target token rotation time set up at system initialization time. The previous result by Johnson and Sercik [26. 40] is a special case where $i=2$. We use this result in the analysis of varions suchronous allocation schemes. It can also be appliel in other similar studies.
\end{abstract}

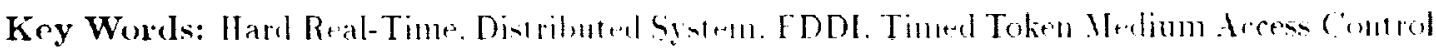

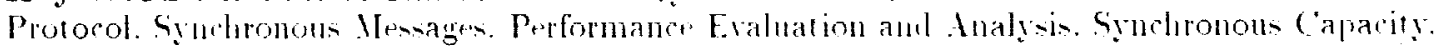
Wors case dichable Crilization.

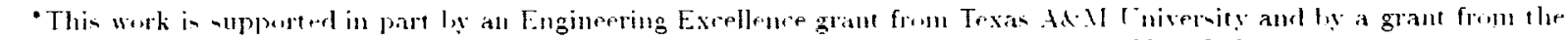

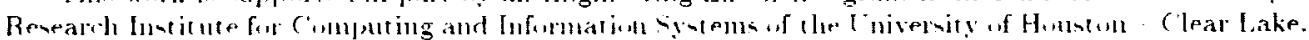




\section{Contents}

1 Introduction 1

2 Previous Relevant Work 3

3 System Characteristics 4

3.1 Vetwork model . . . . . . . . . . . . . . . . . . . . . . 4

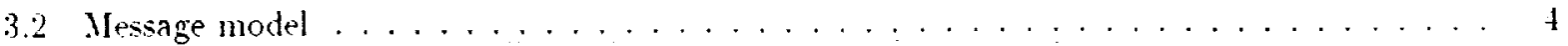

4 Timed Token Medium Access Control Protocol 5

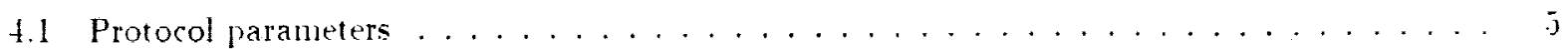

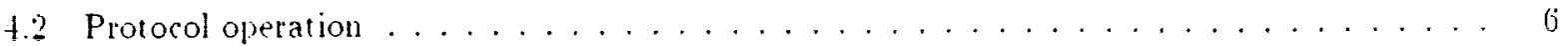

4.3 Synchronous capacity allocation schemes . . . . . . . . . . . T

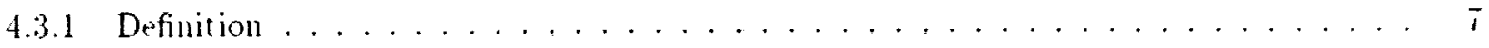

4.3 .2 Requirements . . . . . . . . . . . . . . . . . 8

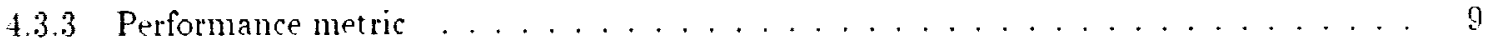

5 Protocol Timing Properties $r$

6 Analysis of Synchronous Capacity Allocation Schemes $\quad 11$

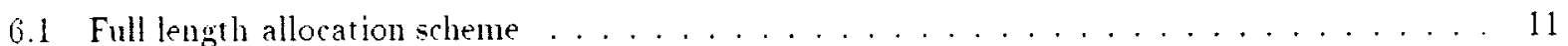

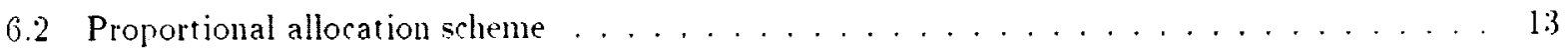

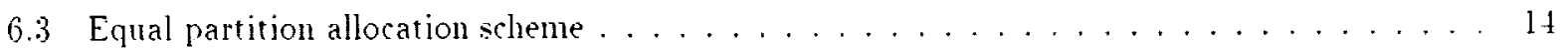

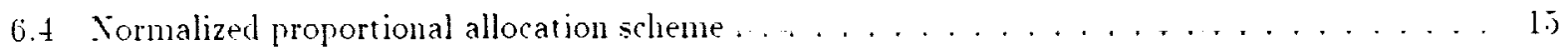

$\begin{array}{llr}7 \text { Conclusion } & 17\end{array}$

8 References $\quad 19$

Appendix A Transformation of Network Model 22

Appendix B Proofs of Protocol Timing Properties 23

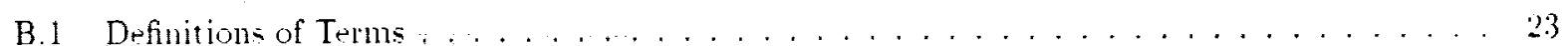

B.2 Proofs of Theorems 5.1 and 5.2 and Corollary $5.1 \ldots \ldots \ldots \ldots \ldots \ldots$

$\begin{array}{lll}\text { Appendix C } & \text { Proofs of Lemma } 6.1 \text { and Theorem } 6.3 & 29\end{array}$

(.1 Proof of Lemmana $6.1 \ldots \ldots \ldots \ldots \ldots$

$\left(\begin{array}{c}2 \\ 2\end{array}\right.$ 


\section{Glossary}

\begin{tabular}{|c|c|c|}
\hline Term & Defintion & Locotion \\
\hline$C_{i}$ & The length (i.e.. transmission time) of a message in synchronous message stream $s_{i}$. & 3.2 . \\
\hline$H_{i}$ & The synchronous capacity allocated to node $i$. & 4.1 \\
\hline$L C_{i}$ & The late counter at node $i$. & 4.1 \\
\hline$\therefore A_{i}$ & The set of asynchronous messages at node $i$. & A. \\
\hline$\therefore S_{i}$ & The set of syuchronous message streams at node; & A. \\
\hline$P_{i}$ & The period length of sinchronous messages stream $s_{i}$. & 3.2 \\
\hline$R_{i}(l)$ & The time at which the token is expected to arrive at node $i$ after its $f^{\text {th }}$ visit at that node. & B. \\
\hline$S_{i}$ & The $j^{\text {th }}$ synchronous message stream at node $i$. & $A$ \\
\hline IH $H T_{i}$ & The token holding timer at node $i$. & 4.1. \\
\hline$T R T_{i}$ & The token rotation timer at node $i$. & 4.1. \\
\hline$T R T_{i}^{\prime}$ & It is defined as being equal to $T R T_{i}+\left(1-L C_{i}\right) \cdot T T R T$. & B. \\
\hline TTRT & The Target Token Rotation Time. & 4.1 \\
\hline$T\left(X_{i}\right)$ & The transformation of node $i$ to a set of virtual nodes. & A. \\
\hline$\varphi(. M)$ & $\begin{array}{l}\text { The utilization factor of the synchronous messages. i.e. fraction of the time spent hy the } \\
\text { network in transmission of the synchronous messages. }\end{array}$ & 3.2. \\
\hline$\tau_{x}^{-}$ & The Achievable Ctilization of sychronous capacity allocation scheme $x$. & 4.3 .3 \\
\hline$\tau_{r}^{*}$ & The Worst Case tchievable Ctilization of suchronous capacity allocation scheme $x$. & 4.3 .3 \\
\hline$Y_{i}$ & The $j^{\text {th }}$ virtual node derived from node $i$ after its transormation. & A. \\
\hline$X_{i}$ & $\begin{array}{l}\text { The amount of time arailable to node ito transmit its sunchronous messages within a given } \\
\text { period. }\end{array}$ & j. \\
\hline$m$ & The number of (virtual) nodes in the lietwork. & 3.1. \\
\hline$n$ & $\begin{array}{l}\text { The number of synchronous message stream in the netrork. In this paper. it is assumed } \\
\text { that } n=m \text {. }\end{array}$ & 3.2 \\
\hline$\theta_{i}$ & The latency between node $i$ and its upstream neighbor. & 3.1 . \\
\hline$\Theta$ & The total ring latency or token walk time. & 3.1 . \\
\hline$\lrcorner$ & The protocol dependent overbeads. & 4.3 .2$. \\
\hline T & The portion of the $T T R T$ that is muavalable to transmit suchronous messages. & 4.3 .2 \\
\hline$n$ & The ratio of t to the Target Token Rotation Time (TTRT). & 4.3 .2 \\
\hline$t_{i}(l)$ & The time when the token arrives at node $i \mathrm{in}$ it $-f^{\text {th }}$ visit. & 5. \\
\hline
\end{tabular}




\section{Introduction}

High sped networks are vital for the support of distributed real-time applications (e.g. voice/video transmission. process control). Dist ributed real-time systems may be categorized as soft real-time systems or hand real-time systems. In sof real-time systems tasks are performed by the system as fast as possible but are not constrained to finish by a specific time. In hard real-time systems tasks must satisfy explicit time constraints: otherwise. grave conscuuences may result. Consequently. the messages transmitted in the network hy the hard real-time tasks are also time constrained. There are two common types of time constraints: laxity. which specifies the maximum time a message can wait before its transmission begins. and deadline. which defines the latest time by which the transmission of the message must finish. In this paper. we address the issue of guaranteeing synchronous message deadlines in high speed networks. By guarantefing. we mean that as long as the network operates normally (i.e.. no failures). synchronous messages are always transmitted before their deadlines.

We address the issue of guaranteeing message deadlines with the timed token medium accos control (1/AC) protocol [1.5]. This protocol is suitable for real-time applications not only because of its use in high bandwidth networks but also due to the fact that it has the import ant property of bounded access time which is necessary for real-time communications. The timed token protocol has been incorporated into many network standards. including the Fiber Distributed Data Interface (FDDI) [1. 2]. IEEE 802.4 [19]. the HighSpeed Data Bus and the High-Speed Ring Bus (HSDB/HSRB) [8. 46. 47. 58]. and the Survivable Adaptable Fiber Optic Embedded Network (SAFEXET) [14. 2\$. 35. 40]. Many embedded real-time applications use them as backbone networks. For example the EDDI has been selected as a backbone network for IASA's Space Station Freedom [4. 7.61$]$.

With the timed token protocol. messages are grouped into two separate classes: the synchronous class and the asynchronous class. Synchronous messages arrive in the srstem at regular intervals and may he associated with deadline constraints. The idea belind the timed token protocol is to control the token rotation time. At network initialization time. a protocol parameter called Target Token Rofation Time (TTRT) is determined which indicates the expected token rotation time. Each station is assigned a fraction of the TTRT. known as synchronous capacity. ${ }^{1}$ which is the maximum time for which a station is permitted to transmit its sychronons messages every time it receives the token. Once a node receives the token. it transmits its sychronous message. if any. for a time no more than its allocated synclironous capacity. It can then transmit its asyncluronous messages only if the time elaped since the previous token departure from the same node is less than the value of ITRT. i.e. only if the token arrived earlier than expected.

Guaranteeing a message deadline implies transmiting llo mesage before its deadline. Wirls a token passing protocol. a node can transmit its message ouly when it captures the coken. This implies that if a message deadline is to be guaranteed. the token should risit the node where the message is waiting before the expiration of the mesages deadline. That is in order fo guarante message deadlines in a token ring

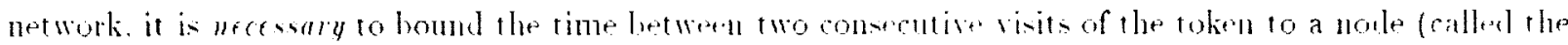

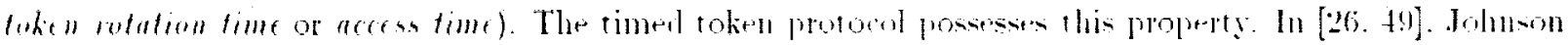
and Sercik formally proved that when the network opetates normally (i.e. there is no failure). 1he twen

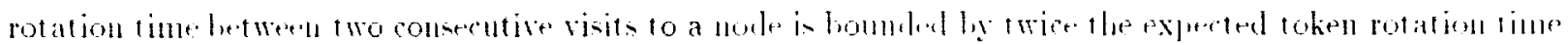

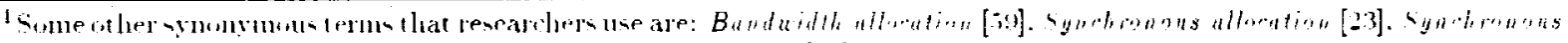

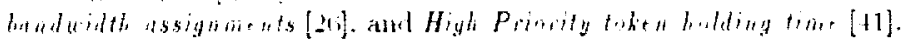


(i.e.. $2 \cdot T T R T$ ).

Although the prerequisite of bounded token rotation time is indispensable. it is insufficient for guaranteing message deadlines. A node with inadequate sychronous capacity may be unalule to complete the transmission of a syuchronous message before its deadline. On the other hand. allocating excess amounts of synchronous capacities to the nodes could increase the token rotation time, which may also canse mesage deadlines to be missed. Thus guarantering message deadlines is also dependent on the appropriate allocation of synclironous capacities to the nodes. As pointed ont in [26]. the allocation of syclironous capacities is an open problem. The main ohjective of this study is to analyze and evaluate the synchronous capacity allocation schemes used with the timed token protocol in a hard real-time communication sistem.

Before discussing details of our work. we will first present an analogy between real-time communication and scheduling to motivate the readers towards the use of our methodology. For real-time systems, the basic design requirements for a communication protocol and for a centralized scheduling algorithm are similar: both are constrained by time to allocate a serially used resource to a set of processes. Liu and Layland [31] addressed the issue of guaranteeing the deadlines of sychronous (i.e. periodic) computation tasks in a single ('PC environment. They analyzed a fixed priority preemptive algorithm. called the rate monotonic algorithm. which assigns priorities to tasks in a reverse order of the task's periods. They showed that the Worst Cast Achic rable Ctilisation of the algorithm is $69 \%$. As long as the utilization of the rask set is no more than $69 \%$, task deadlines are guaranteed to be satisfied. The algorithm was also proven to be optimal among all the fixed priority scheduling algorithms in terms of achieving the highest worst case utilization. The rate monotonic scheduling algorithm has been subsequently extended by many researchers [9. 50] and is used in many hard real-time applications [10].

Int uitively, one rould believe that a communication protocol which implements the rate monotonic transmission policy is the most desirable for a real-time communication environment. However. implementation of the rate monotonic policy requires global priority arbitration every time a node in the network is ready. to transmit a new frame. In a high speed network. such as the FDDI network, where the bandwidth can be as high as 100 Mhps. the orerheads involved in global priority arbitration would be too prohibitive in comparison to the transmission times of the messages themselves. Consequently. it is difficult if not imposible. to implement the rate monotonic transmission policy in such enviromments.

However. the methodology for analyzing this algorithm has a more profound significance than merely its relevance to the rate monotonic scheduling. The methodology stresses the fundamental refuirement of predictability and stability in hard real-time enviromments and is therefore also hefitting to other hard realtime scheduling problems. In this methodology. the Wort Case Achievable Ctilization is useil as a metric for evaluating the predictability of a scheluling algotithm. That is if the (CPI utilization of all task is within the bounds specified ly the metric. all the tasks will meet hlepir deadlines. This metric also gives a measure of the stability of the schechuling algorithm in the sense that the tanks can be freely morlifierl as long as their total utilization is held within the limit. These advantages (of predictahility and stalility) have led un to adopt the same methodology in our study of guarantesing mesage deadlines with the timed token

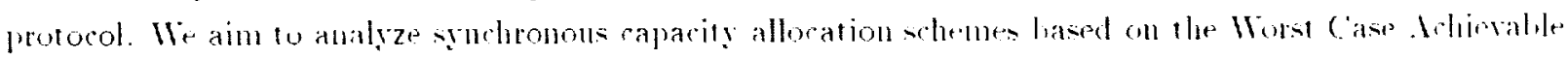
Ciilization.

In this paper. four suchonous rapacity allowarion shemes are antlyzed. Our analysis reveals that

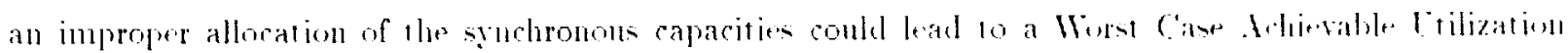


that asmoptotically approaches $0 \%$. That is, the deadlines of some messages could he missed even if the synchronous traffic is arbitrarily close to zero. On the orber hand, one of the schemes proposed in the paper - the normaliod proportional allocation scheme - has a Worst Case Achevahle l'tilization of 33\%. That is. as long as the tot al synchronous traffic is no more than $33 \%$. the synchronons messages are guaranted to be transmitted hefore their deadlines (regardless of the number of stations, message lengths. periods, phases. etc.) The remaining $65 \%$ of the channel capacity could he used ly asynchronous traffic. To the hest of our knowledge no other scheme las been reported to achieve a better utilization. Hence this allocation scheme should be recommended for use in hard real-time communication networks that use the timed token MAC protocol.

Another major contribution of this paper is to extend the analysis of the bound on the token rotation time given by Johnson and Sercik in [26, 4!]. We show that the time elapsed between any $t$ consecutive arrivals of the token at a node is bounded by $x$. TTRT. The previous result by Johnson and Sevcik [26, 49] is a special case where $r=2$. Our newer hound is used in the analysis of the sunchronous capacity allocation schemes and will be applicable in other similar studies.

The remainder of the paper is organized as follows: Section 2 will review the previous relevant work. Section 3 will outline the characteristics of the system under consideration. i.e., the message and network models. The timed token protocol and the synchronous capacity allocation schemes are introduced in Section 4. Section 5 discusses some timing properties of the protocol. In Section 6 we will study several allocation schemes and derive their Worst Case Achievable litizations. Section $\bar{i}$ contains the concluding remarks and suggestions for future work.

\section{Previous Relevant Work}

Extensive research has been done on the timed token protocol since it was first proposed ly (irow [1.j] in 1082. Introductory tutorials on this protocal and its use in networking standards can be found in the papers by Ross [43. 44. 4.5]. Iyer and Joshi [20.21] and others [34, 52. 5.3].

Some important characteristics and architectural design considerations of FDDI token ring networks are

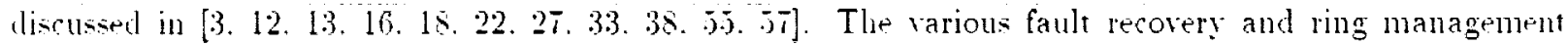
procedures of the FDDI are outlined in the papers by Ocheltree and Montalvo [38. 39]. An orerview of the FDDI MAC services is given in [.57]. Design consirlerations and the role of concentrators are discussed in [1i. 18]. Isstes concerned with interoperahility and interconnection of FDDI with heterogeneous urtworks can be found in $[3,6,16,3: 3]$.

The timing properties of the FDDI token ring were firt formally analyzed by Johnson and Seveik in $[26,49]$. Other interesting timing properties of the FDDI were given in a study conducted hy .Jain [2:3]. IIF slygests that a value of 8 ms for TTRT is desirable a it can achiesp sok urilization on all coufigurations and roult in less than 1 second maxinum acess delay on large rings. Furtlere simulation stulies have leen

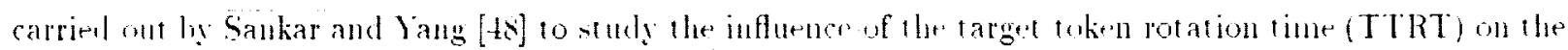
performance of varions FDDI ring configurations.

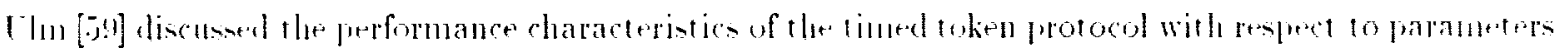


such as the channe] capacily. lhe network cable length. and the mumber of stations. Dykeman and Bux [11] studied and developed a procedure for estimating the maximum throughput of asychronons messages when using single and multiple asychronous priority levels. They aloo proposed a procedure for tuniug the protocol for desired performance hy sefting appropriate values for the token-holding-time thresholds for each of the priority levels. Other analysis concentrating on the performance of the FDDI with respect to the throughput of asynchronous traffic has been done by Pang and Tobagi [41]. Jayasumana and Werahera [24], Valenzo. Mont uschi, and ciminiera [iou. etc.

Note that none of the above studies on the timed token protocol have specifically addressed the use and performance of the protocol in hard real-time environments. On the other hand. many studies of CSMA/C'D and token ring protocols for distributed hard real-time applications have been conducted. The issues in design and analysis of deadline driven communication protocols for CSMA/CD networks are addressed in [5. 29. 32. 42, 51. 56. 63.64.65.66]. The real-time performance of various token ring protocols are considered in [30. 37. 31, 54. 62]. Our work reported in this paper complements the previous studies hy addressing the issues pertinent to hard real-time communication in a high speed network where the timed token medium access control protocol is utilized.

\section{System Characteristics}

In this section an overview of the system under consideration is given. including the network and message models.

\subsection{Network model}

We consider the network topology as consisting of $m$ nodes connected by point-to-point links forming a circle i.e.. the token ring. A special bit pattern called the token circulates around ring (from node $i$ to nodes $i+1$. $i+2, \ldots$ until node $m$. then to nodes $1.2 \ldots$ ). helping to determine which node should send a frame of message among the contending nodes.

We denote the latency between a node $i$ and its upstream neighbor ${ }^{2}$ by $\theta_{i}$. This delay includes the node bit delay, the node latency luffer delay: the media propagation delay. etc. The sum total of all such latencies in the ring is known as the ring latency $\Theta$. i.e. $\sum_{i=1}^{n} \theta_{l}=\Theta$. Thus. the ring latency $\Theta$ denotes the token walk time around the ring when none of the nodes in the network disturl, it.

\subsection{Message model}

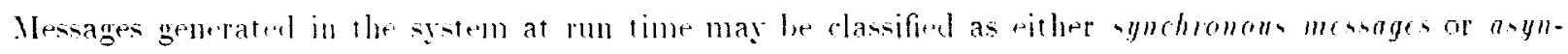

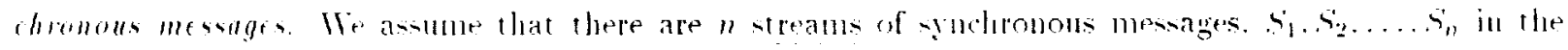
s!st-m which form a syluphronous me wagt wel. W. i...

$$
M=\left\{s_{1}, s_{2} \ldots . . . i_{n}\right\} .
$$

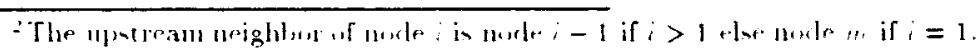


The characteristics of messages are as follows:

1. Synchronons messages are periodic, i.e.. messages in a syuchronous message stream have a constant inter-arrival time. We denote $P_{1}$ to be the period length of strean $S_{i}(i=1.2 \ldots \ldots n)$.

2. The deadline of a syuchronous message is the end of the period in which it arrives. That is if a message in stream $S_{i}$ arrives at time $t$, then its deadline is at time $t+P_{i}$.

3. Messages are independent in that message arrivals do not depend on the initiation or the conpletion of transmission requests for other messages.

4. The length of each message in stream $S_{i}$ is $C$, which is the maximum amount of time needed to transmit this message.

5. Asynchronous messages are non-periodic and do not have a hard real-time deadline requirement.

The utilisation factor of a synclironous message set. $[(M)$, is defined as the fraction of time spent by the network in the transmission of the synchronous messages. That is.

$$
U^{*}(M)=\sum_{i=1}^{n} \frac{C_{i}}{P_{i}}
$$

where $n$ is the number of sychronous message streams.

In the following discussion we assume that there is one stream of synchronous messages on each node (i.e. $m=n$ ). In Appendix $A$. we show that an arbitrary token ring network where a node may have zero. one, or more streams of synchronous messages can be transformed into a logically equivalent network with one stream of stuchronous messages per node. Hence, this assumption of one stream per node simplifies the analysis without loss of generality. We also assume that the network is free from hatdware or sof ware failures.

\section{Timed Token Medium Access Control Protocol}

\subsection{Protocol parameters}

The timed token protocol uses the following parameters and variables for its operation.

1. Target Token Rotation Time (TTRT). When the network is initialized. the value of the TTRT is determined. Which gives the expected value of the token rotation time. It is selected to be sufficiently small to support the response time requirements of the messages at all the nodes in the uetwork. Since the tint "lapserl betwen two consecutive visits of the tuken at a norle can be as much as 2. TTRT [29i]. a node may nut be alle to tmansmit any mescagt in this iuterval.

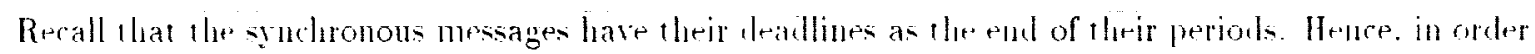

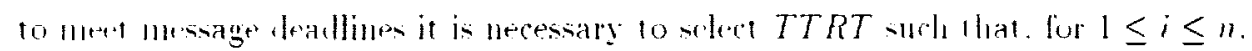

$$
T T R T \leq \frac{P_{i}}{2}
$$


where $P$, is the period of syuchronons message steam $S_{j}$. Any $P$, may therefore be pepresuted as a linear function of TTRT. That is.

$$
P_{i}=m_{i} \cdot T T R T-\dot{c}_{i}
$$

where $m_{i}=\left\lceil\frac{P}{T T R T}\right\rceil \geq 2$. If $m_{i}=2$. then $\delta_{i}=0$ and if $m_{i} \geq 3$ then $0 \leq \delta_{i}<T T R T$. The aline expression for $P_{1}$ has been introduced as it will be useful in several proofs encountered later on. Whe assume that (3) holds throughout this paper.

2. Syuchronous capacity of nodf $i\left(H_{i}\right)$. This paraneter represents the maximum time for which a station is pernitted to transmit sunchronous messages every time the station receives the token. Note that each station can be assigned a different $H_{i}$ value. ${ }^{3}$ This paper will deal with the issue of appropriate allocation of these $H_{i}$ values.

3. Token Rotation Timer of node i (TRTi). This counter is initialized to equal TTRT, and count down until it expires (i.e.. $T R T_{i}=0$ ) or until the token is received and the time elapsed since the previous token departure is less than TTRT. In either situation. the $T R T_{i}$ is reinitialized to TTRT. After being reset. it continues the subsequent counting down cycles in the same manner as above.

4. Token Holding Timer of node $i\left(T H T_{i}\right)$. This (down) counter is used to control the amount of time for which the node $i$ can transmit asynchronous messages.

j. Late Counter of node $i\left(L C_{i}\right)$. This counter is used to record the number of times that TRT, has expired since the last token arrival at node $i$.

\subsection{Protocol operation}

At ring initialization. the following parameters are initialized at all nodes:

1. $T H T_{i}-0$ :

2. $L C ;-0$ :

3. $T R T_{i}-I T R T$.

The TRT, counter always counts down. When it reaches zero. the following acrions take place:

1. $T R T_{i}-T T R T$ :

2. $L C_{i}-L C_{i}+1$.

The TRT, then begins the comting down process again with $L C$, heing incremented by one at every expiration of TRT . Normally. if $L C$, exceeds one. the ring rerovery process is intiated [2.:].

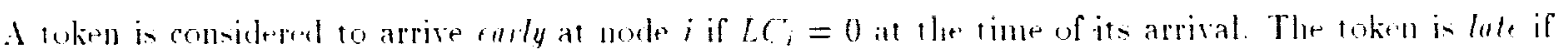
$L C_{i}>0$.

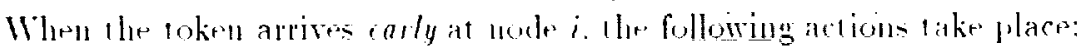

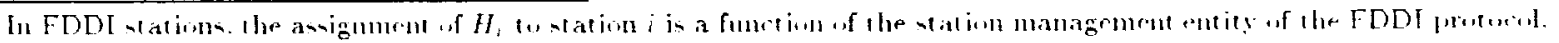


1. $T H T_{i}-T R T_{i}$;

2. $T R T_{i}-T T R T$;

3. Synchronous frames (if any) can then be transmitted for a maximum time of $H_{i}$ (i.e.. the sychronous capacity at node i):

4. After transmitting sychronous frames (if any). the station enables counter $T H T_{i}$ (i.e. it starts counting down). The station may then transmit asynchronous franes as long as $T H T_{i}>0$ and $T R T_{i}>0$.

When the token arrives late at node $i$. the following actions take place:

1. $L C_{i}-0$ :

2. $T R T_{i}$ continues to count down towards expiration. Note that it is not reset to TTRT as in the case when the token is early:

3. Node $i$ can transmit synchronous frames for a maximum time of $H_{i}$ :

4. No asynchronous frame will be transmitted.

Figure 1 shows an example of how $T R T_{i}$ and $L C_{i}$ (at some node $i$ ) vary with time $t$. At point $\mathrm{B}$ in the figure, the node receives the token early. At point $F$. the token is received late. Synchronous messages are transmitted in both cases. but asynchronous messages are transmitted only when the token arrives early.

\subsection{Synchronous capacity allocation schemes}

As mentioned earlier. synchronous capacity allocation plays an important role in guaranteeing syuchronous message deadlines. In this subsection. we formally present the definition of allocation schemes and discuss their requirements and performance metrics.

\subsubsection{Definition}

The synchronous message parameters (given by the $C ;$ s and $P ; s$ ) at the rarious stations and the Target Token Rotation Time (TTRT) should be the dictating factors for the allocation of the $H_{2}$ s. We define a sruchronons capacity allocation scheme as an algorithm which. When given as input the values of all $C$, and $P_{j}$ in the message set and the value of TTRT. will produce as output the values of the symphousts capacities $H_{i}$ to be allocated to station $i$ in the network. Fonmally. It function $f$ represent an allocation scheme. Then.

$$
f\left(C_{1},\left(C_{1} \ldots C_{n}, P_{1}, P_{2} \ldots, P_{n}, T T R T\right)=\left(H_{1}, H_{2} \ldots H_{n}\right)\right.
$$

Let us consider a simple example. We assume a network with only 3 notes. We haw the following values for the message set p paraneters:

$$
\begin{array}{ll}
C_{1}=1 / 2, & P_{1}=1 . \\
C_{2}=1 / 2, & P_{2}=2 . \\
C_{3}=1 / 2 . & P_{3}=2 .
\end{array}
$$


The value of TTRT is assumed to be $1 / 2$. Ising an allocation scheme where

$$
H_{i}=\frac{C_{i}}{P_{i}} \cdot T T R T
$$

we oltain the values of sunchronous capacities as:

$$
\begin{aligned}
& H_{1}=\frac{C_{1}}{P_{1}} \cdot \operatorname{TTRT}=1 / 4, \\
& H_{2}=\frac{C_{2}}{P_{2}} \cdot \operatorname{TTRT}=1 / \mathrm{A} . \\
& H_{3}=\frac{C_{3}}{P_{3}} \cdot \operatorname{TTRT}=1 / \mathrm{R},
\end{aligned}
$$

i.e.. $f\left(C_{1} \cdot C_{2} \cdot C_{3} \cdot P_{1} \cdot P_{2}, P_{3} \cdot T T R T\right)=\left(\frac{1}{4} \cdot \frac{1}{8} \cdot \frac{1}{3}\right)$.

In Section 6. we will introduce several other allocation schemes and analyze their effect on the real-time performance of the network. Before that, we will discuss the general requirements that any allocation scheme should sat isfy.

\subsubsection{Requirements}

The synchronous capacities allocated to the nodes by any scheme must satisfy the two constraints given below in order to ensure that the real-rime messages can be transmitted before their deadlines and that the timed token protocol requirements are satisfied.

- Protocal constraint: Theoretically. the total available time to transmit synchronous messages. during one complete traversal of the token around the ring. can be as mucli as TTRT. However. factors such as ring latency $\Theta$ and other protocol/network dependent overheads reduce the total arailahle time to transmit the synchronous messages. We denote the portion of TTRT unarailable for transmitting synchronous messages by $\tau$. That is $\tau=\Theta+\perp$ where $\perp$ represents the protocol dependent orerlieadls. ${ }^{4}$ We define the ratio of $\tau$ to the target token rotation time (TTRT) to be a. The usable ring utilization arailable for sinchronous messages would therefore be $(1-n)[.5 y]$.

Thus. a protocol constraint on the allocation of synchronous capacities is that the sum total of the synchronous capacities allocated to all nodes in the ring should not be greater than the acalable portion of the Target Token Rotation Time (TTRT) i.e..

$$
\sum_{i=1}^{n} H_{i} \leq T T R T-\tau
$$

- Deadline constraint: The allocation of the symchronous copacities to the medes should le sech that the aynchronous mensages are alerays guaranted to be transmitted hefore the dr deadline a. i.t. le fore the

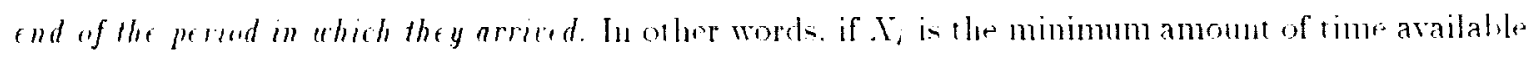

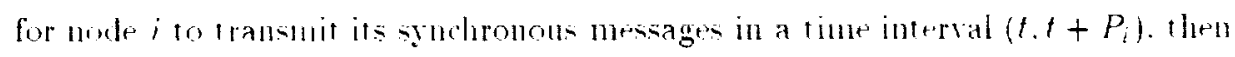

$$
\mathrm{Si}_{i} \geq \mathrm{C}_{i}
$$

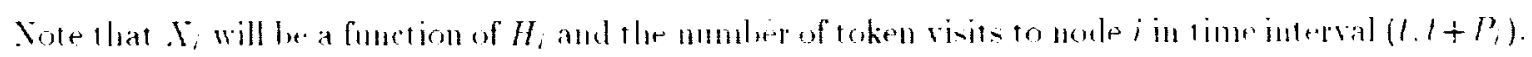

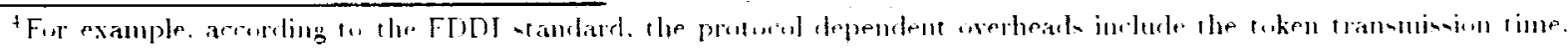

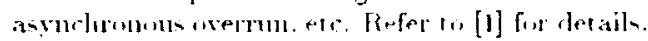


We say a message set is guaranted hy an allocation scheme if hoth the protocol and the dealline con-traints are satisfied. Once a message set is guaranted. messages will be transmitted hefore their deadlines. as long as the network operates normally.

\subsubsection{Performance metric}

Sumerous syuchronous capacity allocation schemes can be proposed. An appropriate meiric is needed in order to evaluate and compare the effects of allocation schemes on the performance of the nemort.

As mentioned in Section 1. we adopt the methodology developed in analyzing the rate monotonic scheduling algorithm. As per this methodology. the Horst Cast Achicrable rthlisation will be used as the metric for evaluating and comparing the allocation schemes.

We say that $t_{r}$ is an Achic cable thlisation of scheme $x$ if scheme $x$ can guarantee erery sychronous

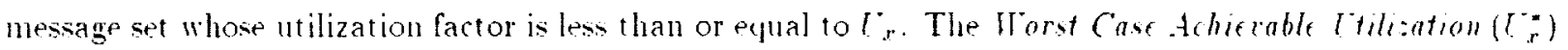
of a scheme $r$ is the least upper hound of its Achievable [tilizations ${ }_{r}^{-} r$. That is as long as the utilization factor of a synchronous message set is no more than $l_{x}^{*}$. the message set can be guaranteed by scheme $x$. In a hard real-time sistem. We consider one scheme to be better than another if its Worst Case Achievable Itilization is higher. When the context is clear, we may omit the index in the notations of $l_{r}^{*}$ and $l_{r}^{*}$.

The major advantages of this metric are as follows:

- This metric evaluates the predictability of a hard real-time communication systems. If the utilization of a suchchonous message set is within the hound specified by the metric all synchronous messages in the set will meet their deadlines.

- This metric also gives a measure of the stability of the system in the seuse that the parameters of synchronous messages can be freely changed as long as their total utilization is held within the limit.

- In practice. using this metric simplifies network management considerably while configuring the sistem. as it elininates the problem of being encumbered with individual values of stuchronous and asynchronous message lengths. inter-arrival periods. phase differences between message arrivals. relative positions of the notes. token position at initialization. etc. As long as the network manager can ensure that the toral utilization of the time-critical synchronous messages is no more than the Whort C'ase Achievable Citilization of the protocol. he/sle can be cognizant of the fact that the message set will be transmitted with no deadlines being missecl.

The objective of this paper is to derive the Worst Case tehievable Ltilization for synchronous capacity allocation xhemes.

\section{Protocol Timing Properties}

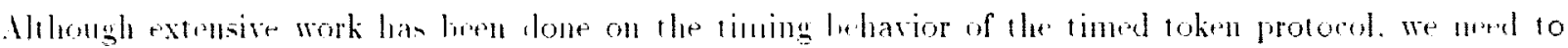

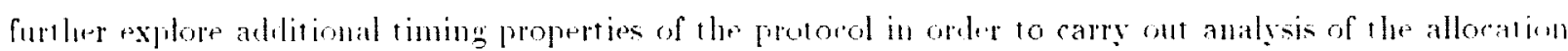

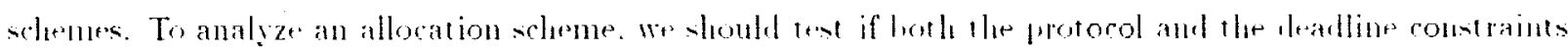


are satisfied. Testing of the deadline constraint is especially clatlenging hecause it involves both network parameters (e.g. $H_{i} . T T R T$, and $\tau$ ) and message parameters (e.g.. $C_{i}$ and $P_{1}$ ). In particular. we need to know the minimum available time (i.e.. the tight lower bound) within a given time period cluring which a node can transmit its sunchronous messages. This is directly related to the minimum mumher (i.e.. the tight lower bound) of token visits to a node within its period.

Johnson and Sercik showed that any two consecutive tokin visits to a node are hounded by 2 . TTRT. Ising this result, we can obtain a lower bound on the minimum number of token visits to a node within the period of its synchronous messages. However. this bound is not tight when the period is longer than 3.TTRT. Because of this. we need to gendralize the analysis done by Johnson and Sercik to oht ain a tiglut bound on the time elapsed between any $x$ consecutive visits by the token 10 a particular node. This then leads us to a derivation of a tight lower bound on the time available for a node to transmit its synchronous messages within a given time period.

Let $t_{i}(l)(l=1.2, \ldots)$ denote the time when the token makes its $l^{\text {th }}$ risit to node $i$.

THEOREM 5.1 (Johnson and Secclis Theorem [-6. 49])

For any integer $1>0$ and any node $i(1 \leq i \leq n)$.

$$
t_{i}(l+1)-t_{i}(l) \leq 2 \cdot T T R T-H_{i} \leq 2 \cdot T T R T
$$

Refer to Appendix B for a proof of the aliove theorem. This theorem gives the upper bound between two consecutive token arrivals as 2.TTRT. A formal proof for the above result was first obtained by Johnson and Sercik in [26. 49]. The tighter upper hound of $\left(2 . T T R T-H_{i}\right)$ will he useful in the analysis of synchronons capacity allocation schemes in Section 6. Text. we will derive a generalized rersion of this theorem.

THEOREM 5.2 (Generalised Johnson and Sercik's Theorem)

For any integerl $>0 . i>0$ and any nodk $i(1 \leq i \leq n)$.

$$
t_{i}(l+r-1)-t_{i}(l) \leq r \cdot T T R T-H_{i}
$$

Refer to Appendix B for a proof of this theorem. This theorem indicates an upper bound on the maximum rime that could possibly elapse between any $x$ consecutive token artivak. Johnon and Sercik : Theorm is a special case when $t=2$. The upper bound specified ly (12) in tight in the semse that he equal sign holls in the worst case situation.

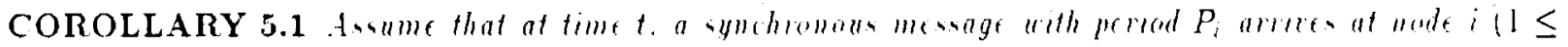

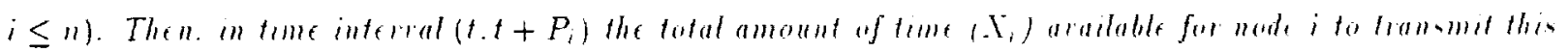
symehremous mesagage in bounded by

$$
K_{i} \geq\left\lfloor\frac{P}{T T R T}-1\right] \cdot H_{i}
$$

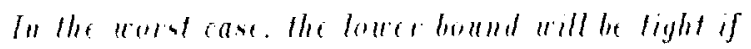

$$
s_{i}=\left\lceil\frac{P_{i}}{T T R T}\right\rceil \cdot T T R T-P_{i} \geq H_{i}
$$


Refer to Appendix B for a proof of the alove corollary. This corollary will he used extensinely in the analysis of our synchronous capacity allocation schemes. Figure 2 shows an example of a worst case scenario where the amount of time for which a node can transmit its sychronons messages is given by the lower bound of (1:3): In the first count down cicle of $T R T_{1}$, the node in the figure toes not receive the token at all. This may happen because some other node may be transmitting its asynchronous messages during this cycte. In the second. the third and the fourth cycles all nodes can transmit only synchronous messages (as the token will visit the nodes late' in these time intervals). In the fifth cycle the node i recejves the tokn too late to transmit its remaining 0.2 units of synchronous messages before the time $t=2.3$. Which happens to be the deadline. That is, node $i$ is able to transmit its synchronous message for 0.6 units of time only: as ran he predicted by Corollary j.1.

\section{Analysis of Synchronous Capacity Allocation Schemes}

In this section we consider four synchronous capacity allocation schemes and derive their Worst Case Achievable Citizations. While the Worst Case Achievable Ctilization of the first two schemes is asymptotically: close to $0 \%$. the third and fourth schemes achieve a non-zero Worst Case Itilization.

We define $P_{\min }=\min \left\{P_{1}, P_{2}, P_{3}, \ldots, P_{n}\right\}$. To simplify our analysis we assume that $P_{m m}$ is normalized to one unit of time. That is all other time variables such as $P_{i}$ s. $C_{i} s . H_{i}$ s. etc.. are measured in this reference time unit.

The underlying principle for computing the Worst Case Achievahle tilization is simple. Given any allocation scheme. we can compute the syuchronous capacity $\left(H_{i}\right)$ arailable to each node $i$. Both protocol and deadline constraints must be satisfied by the allocation of these synchronous capacities. Message sets with the least possible utilization factors are then searched such that the allocation of the sychronous capacities does not satisfy at least one of the constraints. That gives the upper hound on the utilization factor of message sets i.e.. any message set with a utilization factor below that hound will be transmitted successfully without violating either the protocol or the deadline constraints. This then represents the Worst Case tchievable Ctilization of the allocation scheme.

The following lemma will be used in our analysis. Its proof is presented in Appendix $(C$

LEMMA 6.1 For any synchronous mesage tream $i(1 \leq i \leq n)$ we hate

$$
\frac{\left\lfloor\frac{P_{i}}{T T R T}-1\right\rfloor}{P_{i} / T T R T} \geq \frac{1}{3-\frac{i}{T T R T}} \geq \frac{1}{3} .
$$

\subsection{Full length allocation scheme}

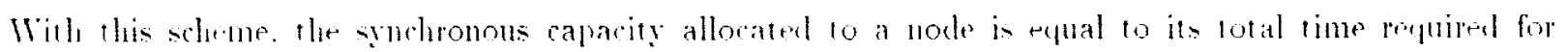
transmitting it - rurluromons messages. i.e.

$$
H_{i}=C_{i}
$$

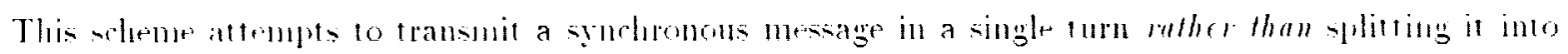

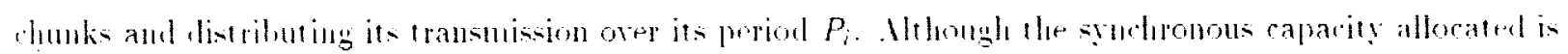


suffirient, dhe Worst Case Achievable Ctilization is zero hecanse the protocol constraint may he violated. as shown in the next theorem.

THEOREM 6.1 The Worst Cose Achir rable Ctiliation of the full length allocation scheme ian anymptetically approach $0 \%$.

Proof: We prove the theorem hy shoring that for any given $\varepsilon>0$. there exists a message set.$M$-urls that $C(M) \leq f$ and the protocol constraint cannot be satisfied when the synchronous capacity of the nodes is allocated using the full length scheme.

Let $T T R T=\frac{1}{k}$ where $k \geq 2$. This is because by (3). TTRT $\leq P_{\min } / 2=1 / 2$. Now, for any given $\epsilon>0$ and $\tau>0$. we construct a set of sinchronous messages as follows:

$$
\begin{array}{ll}
C_{1}=\left(1-\frac{1}{k}\right) \epsilon . & P_{1}=1 . \\
C_{2}=\frac{2-i}{k} . & P_{2}=\frac{2-t}{\epsilon} .
\end{array}
$$

All other $C_{i}=0$ for $i>2$.

The utilization factor is

$$
\begin{aligned}
\tau & =\sum_{i=1}^{n} C_{i} / P_{i}=\frac{\left(1-\frac{1}{k}\right) \epsilon}{1}+\frac{(2-\epsilon) / k}{(2-\epsilon) / \epsilon} \\
& =\epsilon-\frac{\epsilon}{k}+\frac{\epsilon}{k}=\epsilon .
\end{aligned}
$$

With this set of messages. we can show that the protocol constraint is not satisfied. i.e.. the total of all synchronous capacities exceeds TTRT $-\tau$. That is.

$$
\begin{aligned}
\sum_{i=1}^{n} H_{i} & =\sum_{i=1}^{n} C_{i}=C_{1}+C_{2} \\
& =\left(1-\frac{1}{k}\right) \epsilon+\frac{(2-\epsilon)}{h} \\
& =\frac{2}{k}+\epsilon\left(1-\frac{2}{k}\right) .
\end{aligned}
$$

Since $k \geq 2 .\left(1-\frac{2}{k}\right) \geq 0$. Therefore.

$$
\begin{aligned}
\sum_{i=1}^{n} H_{i} & \geq \frac{2}{k} \\
& >\frac{1}{k} \geq \operatorname{TTRT}-\tau
\end{aligned}
$$

Wre see that this scheme may orer-allocate the șinhonous capacity for a message set with milization

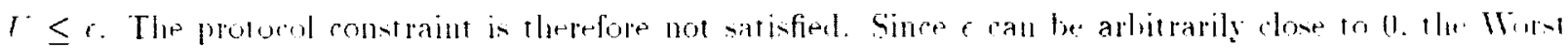

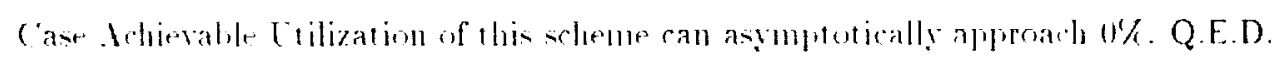




\subsection{Proportional allocation scheme}

Witlit this scheme. the sychronous capacity allocated to a node is proportional to the ratio of $C_{i}$ and $P_{\text {, at }}$ noter i. i.e.,

$$
H_{i}=\frac{C_{i}}{P_{i}} \cdot(T T R T-\tau)
$$

THEOREM 6.2 The Horst Case Achierable Ctilization of the proportional scheme can asymptotically aproash $0 \%$.

Proof: We prove the theorem by showing that for any given $\epsilon>0$. there exists a message set II such that $Y(M) \leq \epsilon$ and the deadline constraint cannot be satisfied when the synchronous capacity of the nodes is allocated using the proportional sclieme.

Let $\operatorname{TTRT}=1 / k$ where $k$ is an integer and $k>2$. Given any $\epsilon>0$. let $\epsilon^{\prime}=\min \left(\epsilon, \frac{1}{2 k}\right)$. Consider a message set with the following parametric values:

$$
\begin{array}{ll}
C_{1}=\left(1-\frac{1}{k}\right) \epsilon^{\prime} . & P_{1}=1 . \\
C_{2}^{\prime}=\left(1+\frac{1}{k}-\epsilon^{\prime}\right) \frac{\epsilon^{\prime}}{k} . & P_{2}=1+\frac{1}{k}-\epsilon^{\prime} .
\end{array}
$$

All other $C_{i}=0$ for $i>2$.

The utilization factor is

$$
\begin{aligned}
\tau & =\frac{C_{1}}{P_{1}}+\frac{C_{2}}{P_{2}} \\
& =\left(\epsilon^{\prime}-\frac{\epsilon^{\prime}}{k}\right)+\frac{\epsilon^{\prime}}{k}=\epsilon^{\prime} \leq \epsilon .
\end{aligned}
$$

The synchronous capacity allocated to node 2 is

$$
H_{2}=\frac{C_{2}}{P_{2}} \cdot(T T R T-\tau) \leq \frac{C_{2}}{P_{2}} \cdot T T R T=\frac{\epsilon^{\prime}}{h^{2}} .
$$

Furthermore. because

$$
\begin{aligned}
\dot{c}_{r_{2}}=\left\lceil\frac{P_{2}}{T T R T}\right\rceil \cdot T T R T-P_{2} & =\left\lceil\frac{1+1 / k-\epsilon^{\prime}}{1 / k^{\prime}}\right\rceil \cdot \frac{1}{k}-\left(1+\frac{1}{k}-\epsilon^{\prime}\right) \\
& =\left\lceil++1-k \epsilon^{\prime}\right\rceil \cdot \frac{1}{k}-1-\frac{1}{k}+\epsilon^{\prime} \quad \text { (since } 0<k \cdot \epsilon^{\prime} \leq 1 / 2 \text { ) } \\
& =1+\frac{1}{k}-1-\frac{1}{k}+\epsilon^{\prime} \\
& =\epsilon^{\prime}>\frac{\epsilon^{\prime}}{k^{\prime 2}}=H_{2}
\end{aligned}
$$

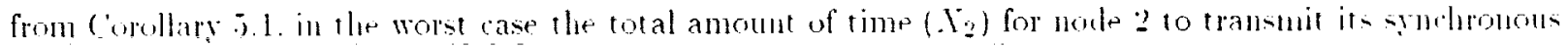
message in a period of $P_{2}$ is giren ly.

$$
\begin{aligned}
I_{2} & =\left\lfloor\frac{P_{2}}{T T R T}-1\right\rfloor \cdot H_{2} \\
& =\left\lfloor\frac{1+1 / k-\epsilon^{\prime}}{1 / k}-1\right\rfloor \cdot \frac{\epsilon^{\prime}}{h^{\prime 2}}=\left\lfloor k+1-k \cdot \epsilon^{\prime}-1\right\rfloor \cdot \frac{\epsilon^{\prime}}{k^{\prime 2}}
\end{aligned}
$$




$$
\begin{aligned}
& =\frac{k-1}{k^{2}} \epsilon^{\prime}=\left(1-\frac{1}{k}\right) \frac{\epsilon^{\prime}}{k} \\
& =\left(1+\frac{1}{k}-\epsilon^{\prime}-\frac{2}{k}+\epsilon^{\prime}\right) \frac{\epsilon^{\prime}}{k}=\left(1+\frac{1}{k}-\epsilon^{\prime}\right) \frac{\epsilon^{\prime}}{k}-\left(\frac{2}{k}-\epsilon^{\prime}\right) \frac{\epsilon^{\prime}}{k} \\
& <\left(1+\frac{1}{k}-\epsilon^{\prime}\right) \frac{\epsilon^{\prime}}{k}=C_{2} .
\end{aligned}
$$

We see that the deadline constraint cannot be satisfied at node 2 . Since 6 can he arbitrarily close to 0 . the Worst Case Achievahle Ctilization of this scheme can asymptotically approach UK. Q.E.D.

Intuitively speaking. this scheme divides the transmission of its message into as many parts as the number of times the token is expected to arrive at node $i$ within its period $P_{i}$. However. since the token could be late by as much as : 2 TTRT. the number of token arrivals is less than expected. Hence. node i may not be alile to complete the transmission of some part of a message before the end of period $P_{i}$.

\subsection{Equal partition allocation scheme}

In this scheme. the usable portion of TTRT is divided equally among the $n$ nodes for allocating their synchronous capacities. i.e..

$$
H_{i}=\frac{T T R T-T}{n} .
$$

where $n$ is the number of nodes in the system.

THEOREM 6.3 The Worst Case Achierable Clilization of the equal partition synchionous capacity allocation scheme is $\frac{1}{3 n-(1-n)} \cdot(1-a)$ where $a=\frac{T}{T T R T}$ and $n$ is the number of nodes.

This theorem can be proved by showing that the following statements are true:

1. For any message set $M$, the protocol constraint will be satisfied.

2. For any message set. II with utilization factor $C^{-}(M) \leq \frac{1-a}{3 n-(1-a)}$, the deadline constraint will be satisfied.

3. For any given $\epsilon>0$. there exists a message set. $I$ with utilization factor $Y(M)=\frac{1-n}{3 n-1|-a|}+\epsilon$. so that the deadline constraint cannot be satisfied for this set of messages when the sinchronous capacities are allocated by using the equal partition scheme.

A detailed proof of this theorem is presented in Appendix $C$.

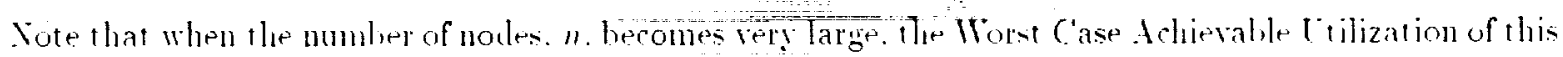
scheme is approximately ok. Intuitively speaking. the for Wont Case Achievahle libation of this sheme

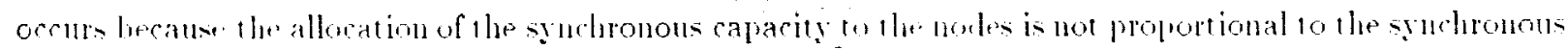

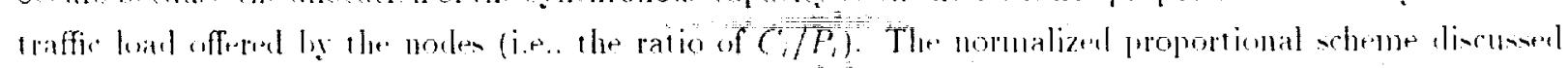

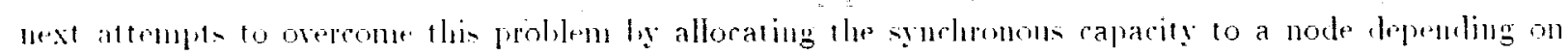

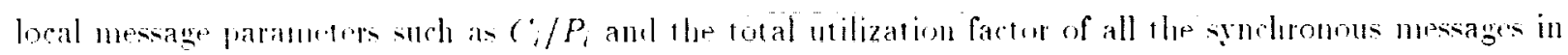
the $-\cdots+m$. 


\subsection{Normalized proportional allocation scheme}

With this sheme. the sychronous capacity is allocated according to the nomalized load of the suchronous message on a node. i.e.

$$
H_{i}=\frac{C_{i} / P_{i}}{I^{-}} \cdot(T T R T-\tau)
$$

where $l^{*}=\sum_{i=1}^{n} C_{i} / P_{i}$

THEOREM 6.4 The ITorst Case Achierable litilisation factor of the normalised proportional allocation scheme is $\frac{1}{3}(1-a)$ uhere $a=\frac{T}{T T R T}$.

Proof: To prove the theorem. we show that the following statements are true:

1. For any message set.$M$, the protocol constraint will be satisfied if $\sum_{i=1}^{n} \frac{C_{P}}{P_{i}}=I^{*} \leq 1$.

2. For any message set.$/$ with utilization factor $C(. M) \leq \frac{1}{3}(1-a)$. the deadline constraint will always be satisfied.

3. For any given $\epsilon>0$. there exists a message set $M$ with utilization factor $\frac{1}{3}(1-a)<U(M) \leq \frac{1}{3}(1-a)+\epsilon$ so that the deadline constraint cannot be satisfied for this set of messages when the synchronous capacities are allocated using the normalized proportional scheme.

Proof of Statement 1: For any message set M with $\sum_{i=1}^{n} \frac{C_{P}}{P_{1}}=l^{*} \leq 1$.

$$
\sum_{i=1}^{n} H_{i}=\sum_{i=1}^{n} \frac{C_{i} / P_{i}}{l} \cdot(T T R T-\tau)=T T R T-\tau .
$$

Hence, the protocol constraint (9) will be satisfied.

Proof of Statement 2: Consider a message set whose utilization factor $T^{-}(. M) \leq \frac{1}{3}(1-a)$. From Lemma 6.1. we have

$$
\begin{aligned}
\ell \leq \frac{1}{3}(1-a) & \leq \frac{\left\lfloor\frac{P_{L}}{T T R T}-1\right\rfloor}{P_{i} / T T R T}(1-a) \\
& \leq \frac{\left\lfloor\frac{P}{T T R T}-1\right\rfloor}{P_{i}}(T T R T-7) .
\end{aligned}
$$

Multiplying with $C / / C^{\circ}$ on hath sides. we get

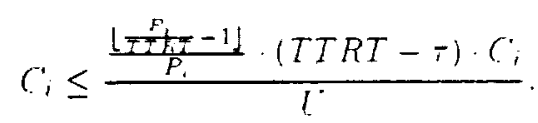

That is. for $1 \leq i \leq n$.

$$
c_{i} \leq\left\lfloor\frac{P_{i}}{T T R T}-1\right\rfloor \cdot \frac{C_{i}}{P_{i} C^{2}} \cdot(T T R T-i)
$$

Sulmiluing $\frac{\text { IP }}{l} \cdot(T T R T-\tau)=H_{i}$, we have

$$
c \leq\left\lfloor\frac{P_{i}}{T T R T}-1\right\rfloor \cdot H_{r}
$$


From Corollary 5.1 and (32), we see that any node $i$ can thansmit it syuch ronous message hefore the tearlline.

Proof of Statement 3: For any given $\epsilon>0$. let

$$
\epsilon^{\prime}=\min \left(\frac{1-a}{3} \cdot \epsilon\right)
$$

where $a=\frac{T}{T T K T}$. Let $T T R T=\frac{1}{2}$. Consider the following message set:

$$
\begin{array}{ll}
C_{1}=\epsilon^{\prime}, & P_{1}=1 . \\
C_{2}=\epsilon^{\prime}, & P_{2}=\frac{3}{2}-\epsilon^{\prime} . \\
C_{3}=1-3 \epsilon^{\prime}-a, & P_{3}=3 .
\end{array}
$$

Sote that Equation (33) guarantes that $C_{3} \geq 0$. All other $C_{;}=0$ for $i>3$.

The utilization of this message set is

$$
\begin{aligned}
C^{*} & =\frac{C_{1}}{P_{1}}+\frac{C_{2}^{\prime}}{P_{2}}+\frac{C_{3}}{P_{3}} \\
& =\epsilon^{\prime}+\frac{\epsilon^{\prime}}{(3 / 2)-\epsilon^{\prime}}+\frac{1}{3}-\epsilon^{\prime}-\frac{1}{3} a \\
& =\frac{1}{3}(1-a)+\frac{\epsilon^{\prime}}{(3 / 2)-\epsilon^{\prime}} .
\end{aligned}
$$

Since $\frac{3}{2}-\epsilon^{\prime}>1$ and $\epsilon^{\prime} \leq \epsilon$. we have

$$
\tau<\frac{1}{3}(1-a)+\epsilon^{\prime} \leq \frac{1}{3}(1-a)+\epsilon .
$$

Consider the synchronous capacity allocated to node 2 :

$$
\begin{aligned}
& H_{2}=\frac{C_{2}}{P_{2} \cdot l} \cdot(T T R T-\tau)=\frac{C_{2}}{P_{2} \cdot l} \cdot \operatorname{TT} R T \cdot(1-0) \\
& =C_{2} \cdot \frac{\frac{1}{2}(1-a)}{\left(\frac{3}{2}-\epsilon^{\prime}\right)\left(\frac{1}{3}(1-a)+\frac{\epsilon^{\prime}}{\frac{3}{2}-\epsilon^{\prime}}\right)}=C_{2} \cdot \frac{\frac{1}{2}(1-a)}{\frac{1}{2}(1-a)-\frac{1}{3} \epsilon^{\prime}(1-a)+\epsilon^{\prime}} . \\
& =C_{2} \cdot \frac{1}{1-\frac{2}{3} \epsilon^{\prime}+\frac{2 \epsilon^{\prime}}{1 !-2)}}=C_{2} \cdot \frac{1}{1+\frac{2 \epsilon^{\prime}}{1-0 !}\left(1-\frac{1-a}{3}\right)} \text {. }
\end{aligned}
$$

Since $0<\epsilon^{\prime} \leq \frac{1}{3}$ and $0 \leq a<1$. the denominator of Equarion (3i) is greater than 1 . Hence.

$$
H_{2}<C_{2}
$$

Wr. now show that $\varepsilon_{2} \geq H_{2}$. We have

$$
\begin{aligned}
8_{2} & =\left\lceil\frac{P_{3}}{T T R T}\right\rceil \cdot T T R T-P_{z} \\
& =\left\lceil\frac{3 / 2-\epsilon^{\prime}}{1 / 2}\right\rceil \cdot \frac{1}{2}-\left(\frac{3}{2}-\epsilon^{\prime}\right)=\left\lceil 3-2 t^{\prime}\right\rceil \cdot \frac{1}{2}-\frac{3}{2}+\epsilon^{\prime} \\
& =\frac{3}{2}-\frac{3}{2}+\epsilon^{\prime} \\
& =\epsilon^{\prime}=C_{2}>H_{2} .
\end{aligned}
$$




\begin{tabular}{|c|c|c|c|}
\hline Vame & Formula of $H_{i}$ & IIC.C.1.: & Comments \\
\hline Full length & $H_{i}=C_{i}^{\prime}$ & 0 & Uses local information only i.e.. $C_{i}$. \\
\hline Proportional & $H_{i}=\frac{\ddot{\ddot{P}}}{P_{1}} \cdot(T T R T-\tau)$ & 0 & Cses local information only: i.e.. $\frac{\mathscr{C}_{\vec{P}}}{\text {. }}$ \\
\hline $\begin{array}{l}\text { Equal parti- } \\
\text { tion }\end{array}$ & $H_{i}=\frac{T T R T-\tau}{n}$ & $\frac{1-a}{3 n-(1-n)}$ & $\begin{array}{l}\text { L'ses glohal information only, i.e. the } \\
\text { number of nodes } n \text {. }\end{array}$ \\
\hline $\begin{array}{l}\text { Normalized } \\
\text { proportional }\end{array}$ & $H_{i}=\frac{C_{i} / P_{1}}{l} \cdot(T T R T-\tau)$ & $\frac{1-a}{3}$ & $\begin{array}{l}\text { [ses hoth local and global information. } \\
\text { i.e. load on the sistem }\left(C^{\circ}\right) \text { and the load } \\
\text { offered by local message streams }\left(\frac{C_{A}}{P^{\prime}}\right) \text {. }\end{array}$ \\
\hline
\end{tabular}

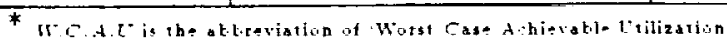

Table 1: Summary of the synchronous capacity allocation schemes.

From Corollary 5.1. the amount of time $\left(X_{2}\right)$ for node 2 to transmit its syuchronous message in a time iuterval $\left(t . t+P_{2}\right)$ is given by

$$
\begin{aligned}
X_{2} & =\left\lfloor\frac{P_{2}}{T T R T}-1\right\rfloor \cdot H_{2} \\
& =\left\lfloor\frac{(3 / 2)-\epsilon^{\prime}}{1 / 2}-1\right\rfloor \cdot H_{2} \\
& =1 \cdot H_{2}<C_{2} .
\end{aligned}
$$

Therefore, the deadline constraint $(10)$ is violated and this set of messages cannot be guaranteed. Q.E.D.

In the normalized proportional allocation scheme. both local information (i.e.. $C_{i}$ and $P_{i}$ ) and global information (i.e., $($ and TTRT) are used. It results in a normalization of the allocated synchronous capacities. therehy achieving a Worst Case tchievable [rilization equal to 33 \% of the available ring utilization.

\section{Conclusion}

Guaranteeing message deadlines is a key issue in distributed real-time applications. The property of tle bounded token rotation time of the timed token protocol provisles a necessary condition to ensure that the message deadlines are satisfied. However the synchronous capacity allocated to each nocle in the network was also shown to be a decisive factor in guaranteeng time-critical mesages. In thi paper. we first derived a generalized rersion of Johnson and Sercik's theorem [26. 49] which gives the maximum time that ran elapse between any ic consecutive token arrivals at onme node. We then applied this result to the andlysis of sychronous capacity allocation shemes. The Wort Case trhiprahle Crilization was used as the metrir to evaluate and compare varions allocation ochemes. This metric is of importance to peal-time applications

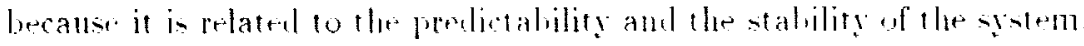

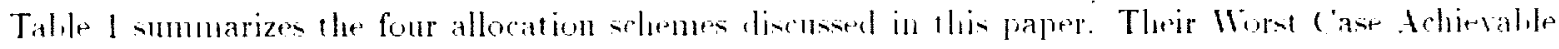

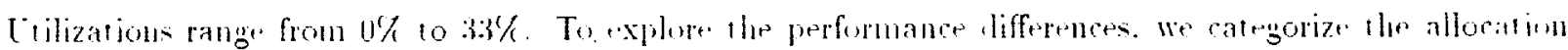
shemes hased on dye type of information they use. An allocation scheme is local if it compunes the syn-

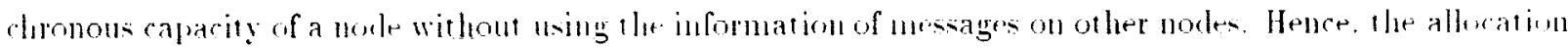


function of a local scheme has the form

$$
H_{i}=f\left(C_{i}, P_{1}, T T R T\right)
$$

On the other hand. a global scheme utilizes system wide information. including the message periods and lengtls on different nodes. the total utilization, the total number of message streams, etc.

As the global allocation schemes use system wide information to allocate synchronous capacitirs. they can reasonahly be expected to result in a better performance than local schemes. Indeed. two glohal schemes proposed in this paper achieve better performance than the local ones as shown in Table 1 . In particular, the nomalized proportional scheme has a high Worst Case dchievable Crilization of $\frac{1-a}{3}$ which is independent of the number of the nodes in the sistem or the message lengths and periods. To date no synchronous capacity allocation scheme has been reported to achieve such substantial performance.

However. it is not get known if the $333 \%$ Worst Case Achievable Ctilization is the highest. This raisps the issue of the optimality of allocation schemes. An optimal allocation scheme should always guarantee a message set if there exists another scheme which can to so. Clearly the optimal scheme has the highest Worst Case Achievable [tilization. Since the global allocation schemes use system wide information. it is likely that an optimal allocation scheme will be a global one. Work is underway to investigate the design and implementation of such an optimal sunchronous capacity allocation scheme.

However. a disadvantage of the glohal schemes lies in the assumption that the message parameters remain constant. A change in a message stream at a particular node may require a re-adjustment of sinchronous capacities over the entire network. This may not be acceptable in some situations. Because local schemes compute the synchronous capacity of a node indepentently of the message parameters at other nodes. they can overcome the above problem. If the parameters of a message stream at a node change during run-time, a local allocation scheme needs to adjust the sunchronous capacity of only the node involved. Orher nodes are not disturbed. That is the entire network can continue its normal operations while individual nodes change their sychronous capacities in response to the changing message parameters. This of course. assumes that the total utilization factor of the message set remains within the Worst Case Achierable Ctilization of the allocation scheme.

Howerer. as the loral allocation schemes use less information than the glohal ones. they may not achiere a Worst Case Achievable Ctilization as high as some of the glohal ones. Both the local allocation schemes

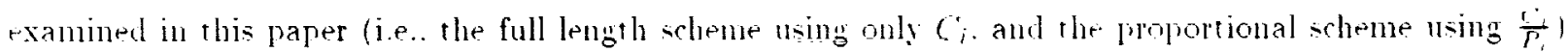
turned out to have a Wors Case Achievable Ctilization of u\%. The prohem therefore remains either to derelop a local allocation scheme with non-zero Wors Cas Achiralife Crilization or to formally prove that all local allocation schemes have a zero Wont Case . hiverahle Itilization. This issue is currenty under investigation.

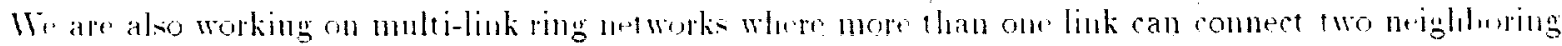
nodes. Will this topology. We would like to stuly the protocol performance in the context of the Wist Cass Achevalum Tilization. 


\section{References}

[1] FDDI Token ring media access control (MAC). ANSI Standard X3.139. 198i

[2] FDDI Token ring station management draft proposed - A.SSI Standard X3T9.5 rev. 5. Ma: 10. 1980.

[3] D. Benwon. P. Ciarfella and P. Hayden. "FDDI: Neeting the interoperability challenge." Proc. IE.EE Conf. on Local Computer Yetworks. pp. 2.33-260, Mimneapolis MX. Sept. 30 - Oct. 3. 1990.

[1] L. Bergman, "Optical protocols for adsanced spacecraft networks." Proceedings of the space station evolution symposium. Vol. 1. part 2. League city. Texas. Aug. 6-8. 1991.

[5] L. X. Bhuyan. D. Chosal and Q. Yang. "Approximate analysis of single and nultiple ring networks." IEEE Transactions on Computers. Vol. 38. To. 7. pp 1027-1040. July 1989.

[6] K. Chap and A. A. Nilsson. "Performance evaluation of FDDI net work and interconnected heterogeneous networks," Proc. IEEE Conf on Local Computer Yetworks pp. 7.j-83. Minneapolis MN. Sept 30 - Oct. 3. 1990 .

[i] E. Cherers, "Adranced DMS architectures." Proceedings of the space station evolution șmposium. Vol. 1. part 2. League city. Texas. Aug. 6-8. 1991.

[8] II. D. Cohn, "A network architecture for advanced aircraft." Proc. IEEE Conf. on Local Computer Yetworks pp. 3.58-364. Minneapolis MIN. Oct. 10-12. 1989.

[0] S. Darari and S. Dhall. "An on-line algorithm for real-time tasks allocation." Proc. IEEE Real-Time Srotems Srmpowium. pp 194-200. Der. 1986.

[10] S. Darari and IV. Zhao. "RISS aids real-time scheduling." RICIS Review. Vol. 3. No. 1. 1991.

[11] D. Dikeman and W. Bux. "Analysis and tuning of the FDDI media access control protocol." Journal of Selected trea in Communications. Vol. 6. No. 6. July 1988.

[12] S. Ginzburg. IT. Mallard and D. Sewman. "FDDI over unshielded twisted pairs." Proc. IEEE Conf on Local Computer Yetrorks. pp. 39.5-398. Minneapolis MX. Sept. 30 - Oct. 3. 1990.

[13] P. Gonia and M. Agrawal. "A high performance OSI implementation on FDDI." Proc. IEEE Conf. on Local Computer Vetrrorks. pp. 301-309. Minneapolis MX. Sept. 30- Oct. 3. 1990.

[14] D. I. Green and D. T. Marlow. "SAFEXET - A LAN for nary mission critical systems." Proc. 14th Conf on Local Computer Tetworks. Minneapolis. IIN. pp. 340-346. Oct. 1989.

[15] R. M. Grow, "A timed token protocol for local area networks." Proc. Electro/82. Token Access Protocols. May 1902.

[16] R. M. Grow. "FDDI follow-on status," Proc. IEEE Conf. on Local Computer Yetworks pp. 4i-48. Mimeapolis MX. Sept. 30-Oct. 3. 1990.

[1i] E. Hotard. "FDDI concentrator design issues." Proc. IEEE Conf on Local Computer Vetworks. pP. $388-394$. Minneapolis .IX. Sppt. 30 - Oct. 3. 1990.

[18] J. Hutchison. "The role of concentrators in FDDI rings" Proc. IEEE Conf on Local Computer Yetworks. p]. 24-40. Minneapolis MI. Oct. 10-12.1989.

[19] [EEE/ANST Standard \$02.t - 105.j "Token passing bus access method and phrsical laver specifications." The Institute of Electrical and Electronic Engineers. Inc.. Sew Jork, 198.3.

[20] V. Iver and S. P. Joshi. "New standards for local networks push upper limits for lightwave data." Data communications.. pp. 12-i-138. July 1984.

[21] Y. Iyer and S. P. Joshi. "FDDI's $100 \mathrm{M}$-hps protocol inproves on \$02.5 Spec's 4 -MI-bps limit." Electrical Design Yers . pp 1.j1-160. Vay 2. 198j.

[2\%] R. Jain. "Error characteristics of fibre clistrihuted lata interface (FDDI)." IEEE Trans on Commun.. Vol 35. No.s. Ang. 1090.

[2:3] R. Jain. "Performance analysis of FDDI token ring netrorks: effect of paraneters and guidelines for setting TTRT." IEEE ITS. pr. 16-2.2. Nay 1991

[2-4] A. P. Jayasmmana and P. X. Werahera. "Performance of filde distributed data interface network for

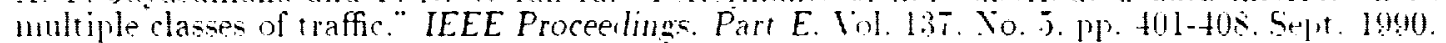

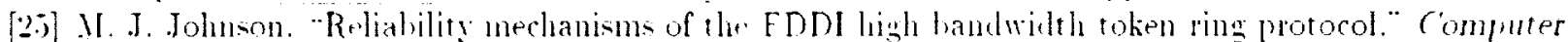

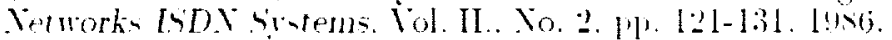

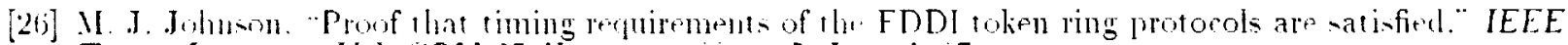

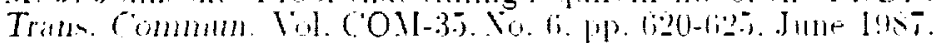

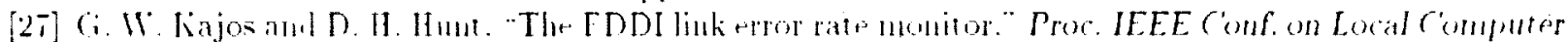

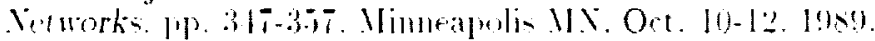

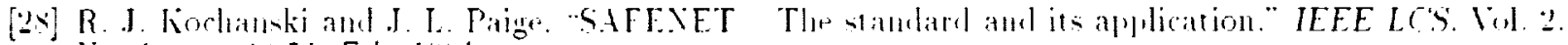

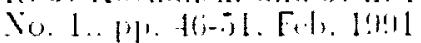

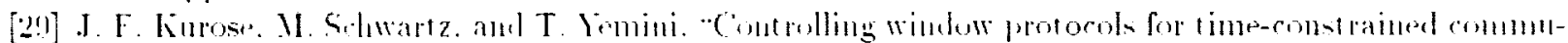

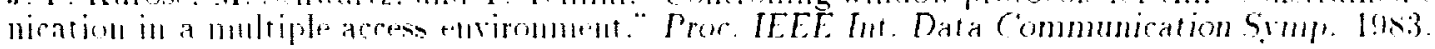


[30] C. C. Lim. L. Yao, and W. Zlao. "A comparative study of three token ring protocols for real-1ime

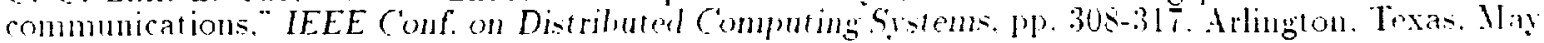
1901 .

[31] C. L. Liu and J. W. Layland. Scheduling algoritlms for multiprogramming in a hard real time environment." J. AC.M. Fol. 20 . Yo. 1. 1973. pp. $16-6 \mathrm{~L}$.

[3:] X. Malcolm and II. Zhao. "Tersion setection schemes for hard pal-time communications." Proc. IEEE Real-time Sistems S!mposium. San Antonio. TX. Dec.. 19!1.

[3:3] P. Martini ant T. Meuser, "Service integration in EDDI." Proc. IEEE Conf on Local Computer Met-

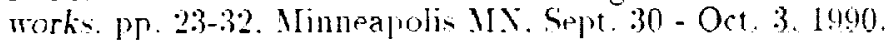

[34] J. Mrcool. “FDDI - getting to the inside of the ring." Data Commun. pl. 18.j-192. March loss.

[3.5] MIL-HDBK-0030, "Survivable adaprable fibre optic embedded network II-SAFE.CET II." Draft.

[36] A. K. Mok and M. L. Dertouzos, "Multiprocessor scheduling in a hard real-time enviromment". Proc. Texas Conf. Computing Sist. Nov. 1978

[3i] J. Sg and J. Liu. "Performance of local area nefwork protocols for hard real-time applications." IEEE Conf. on Distrihuted Computing Systems, pp. 318-326. Arlington. Texas. May 1991.

[38] K. B. Ocheltree and R. M. Montalvo. "FDDI ring management," Proc. IEEE Conf on Local Computer Vetworks pp. 18-23. Mimneapolis MIX. Oct 10-12. 1989.

[39] K. B. Ocheltree. "["sing redundaucr in FDDI networks." Pror. IEEE Conf. on Local Computer Yetworks. pp. 261-267. Minneapolis .IIS. Sept. 30 - Oct. 3. 1690.

[40] Lt. J. L. Paige, "SAFEXET - A nary approach to computer networking." Proc. IEEE Conf. on Local Computer Vetworks. pp. 268-273. Minneapolis .MX. Sept. 30 - Oct 3. 1990.

[H1] J. Pang and F. A. Tobagi. Throughput analysis of a timer controlled token passing protocol under heary load." IEEE Trans on Communications. Vol. 3i. No. T. pp. 694-i02. July 1980.

[42] K. Ramamritham. "Channel characteristic in local area hard real-time sistem." Computer Metworks and ISD.I Sist. Vol. 13. To. 1. Jan. 1985.

[4.3] F. E. Ross. "FDDI-A tutorial." IEEE Commun Mag. Vol. 24. Yo. 5. pp. 10-17. 1986.

[H4] F. E. Ross. "Rings are round for good." IEEE Yetwork Mag.. Jan. $198 \overline{6}$.

[45] F. E. Ross. "An overview of FDDI: The fibre distributed data interface." IEEE Journal on Sel. Areas in Comm. pp.1043-10.51. Vol. T. Sept. 1989.

[46] SAE. Atrospace Srstems Division. Conmittee AS-2. "Linear token-passing multiple data bus." AS4074.1. Version 4.0. Jan. 2.5. 1988.

[ti] SAE. Aerospace Systems Division. Committee AS-2. "High speed ring bus (HSRB)." ASt0it.2. Jan. 2-i. 1988.

[48] R. Sankar and Y. Y. Yang. "Performance analysis of FDDI." Proc. IEEE Conf on Local Computer Tetworks pp. 328-332. Minmeapolis MIX. Oct. 10-12.1989.

[49] K. C. Sercik and .I. J. Johnson. "Crcle time properties of the FDDI token ring protocol." IEEE trans. Soft ware Eng. Vol. SE-13. No. 3. PR. 376-38.3. 1987

[50] L. Sha and J. B. Goodenough. "Real-time scheduling theory and Ada*". IEEE Computer. April 1090.. P). $5.3-62$

[51] K. G. Shin and C. Hou. "Aualytic evaluation of contention protocols used for real-time srstems." Proc. IEEE Real-Time Sirstems Srmp.. Dec 1090

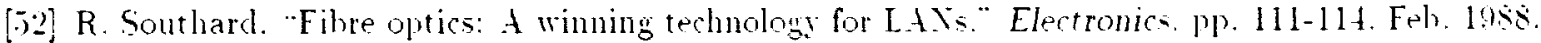

[5.3] WI Stallings. "Computer Communication Stanklards. Fol 2: Local area Vetwork standards." Howard II. Sams (Co.. 19:T.

[;4] J. K. Strosnider. J. Leloczky and L. Sha "Atranced real-time ochecluling using the IEEE s0.2.j token

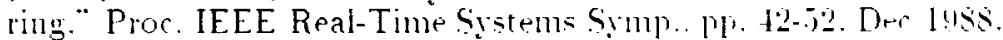

[.5.) .I. Teener and R. (ivozdanovic. "FDDI-II opestation and architectures." Proc. IEEE conf on Local

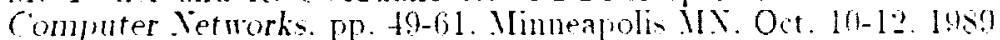

[.56] F. A. Tologai. "Multiaccess protorols and real-fine communications in local area networks." Livanced Sem. Real-time Loral Area Nerwork. Bandol. France. Apr. lii-1s. l!sifi.

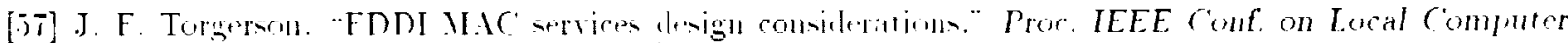

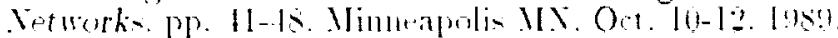

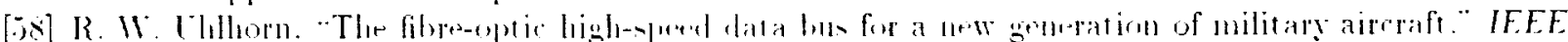

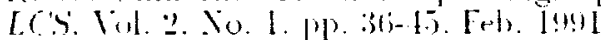

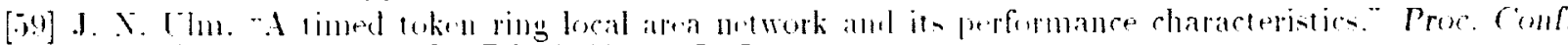

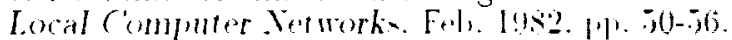

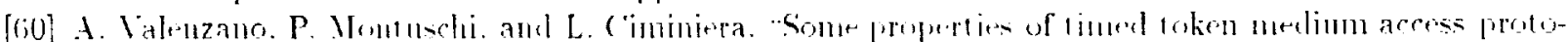

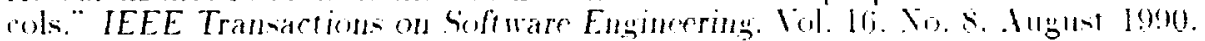


[ij1] A. C. Weaver and R. Sinnoncic. "Communications for the XASA space station," Proc. IEEE ronf on

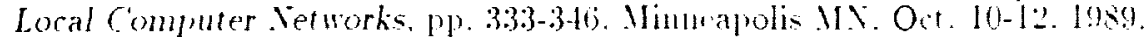

[62] L. Yao and $\mathbb{W}$. Zhao. "Performance of an extended IEEE sol.5 protocol in hard real-time sistems." Proc. IEEE Conf Computer Communications. INFOC O.M 91. A pril 1991.

[6:3] IV. Zhao and K. Ramamritham. "Tirtual time (SUA protocols for hard real-time communications." IEEE Transactions on Software Engineering. SE-13(8):938-9;,2. Aug 198-

[64] W. Zliao. J. A. Stankovic and $\mathrm{K}$. Ramamritham. "A multi-access window protocol for tine comstrained communications." Proc. IEEE Conf. on Distriluted Computing Sistems. June 19s8.

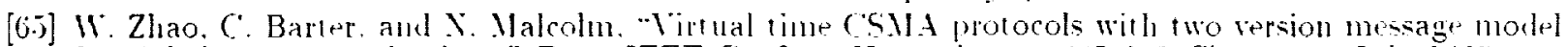
for real-time communications." Proc. IEEE Conf. on Vetworks pp. 29.5-300. Singapore. July j!S!.

[606] II. Zhao. J. A. Stankovic and $K$. Ramamrithan. "A window protocol for transmission of time constrained messages," IEEE Trans. Computers, 39(9):1180-1203. Sept 1090. 


\section{Appendix A Transformation of Network Model}

In this appendix. we present a transformation that converts an arbitrary network model to a logically equivalent virtual model where each node has exactly one sinchronous message stream.

Let node $i$ be demoted hy $Y_{i}$. Zero. one or more synchronous message streams may be arriving at the noth from the external world refuesting transmission. Let the set of sychronous message streans arriving at $V_{i}$ be denoted by $Y_{S} S_{i}$. Hence, if node $V_{i}$ has $l$ streams of synchronous message streams arriving at it. we denote the synchronous message set as:

$$
X S_{1}=\left\{S_{1}, S_{i_{2}} \ldots S_{i_{p}}\right\}
$$

Similarly: the asynchronous message set at node $i$ is denoted as.$A_{i}$. Thus. we can represent node $i$ as:

$$
Y_{i}=\left(X S_{i} \cdot X \cdot A_{i} \cdot \theta_{i}\right)
$$

where $\theta_{i}$ is the latency between node $i$ and its upstream neighlor.

Tode $Y_{i}$ is considered an actirt node if $Y_{S} \neq 0$. That is. there is at least one stream of symchronous messages arriving at node $Y_{i}$. If $Y_{s}=0$. node $Y_{i}$ is an imactice node.

The network can then be represented by the set of nodes as shown below:

$$
\text { Network }=\left\{x_{1}, x_{1} \ldots \ldots x_{m}\right\}
$$

In order to simplify our analysis. the above network model needs to be transformed into a simpler cirtual netuork model in which each virtual node will have one synchronous message stream arriving at it. The transformation. $T$. may be represented as follows:

For all nodes $x_{i}(1 \leq i \leq m)$ in the neturork. do:

- If $x_{i}$ is an actire node with $p$ streams of synchronous messages. It is tranoformed into le cirtual nodes a.s follous:

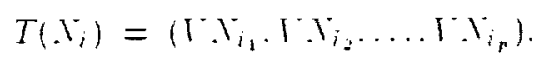

where the rintual node $\mathrm{T}^{2} \mathrm{Y}_{i}$, is represemted as

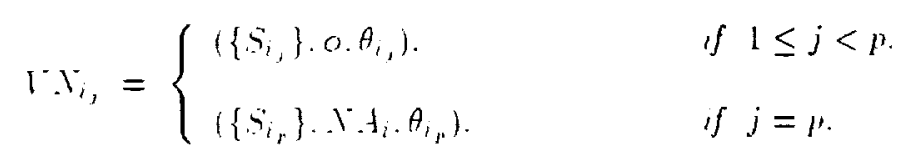

whert

$$
\theta_{i}= \begin{cases}\theta_{i} . & \text { if } j=1 . \\ 0 . & \text { if }: \leq j \leq \mu .\end{cases}
$$

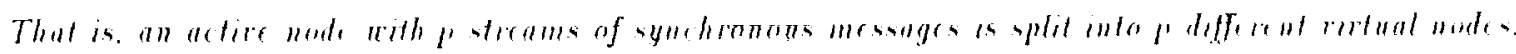

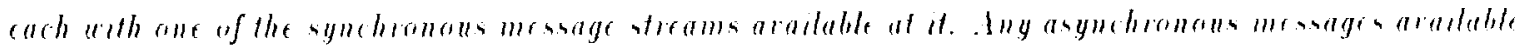

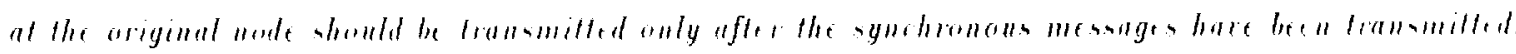




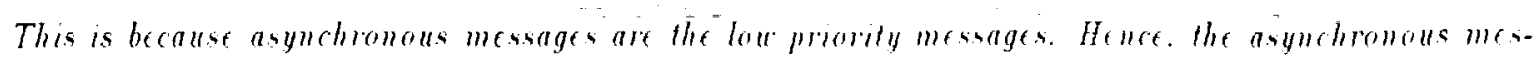
sages at pode $i$ will be consadered to be arailable only at the lant rirtual node ( $\left(x_{i_{p}}\right.$ ) in the doum.tenk

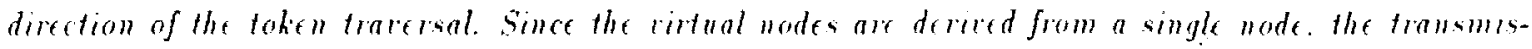

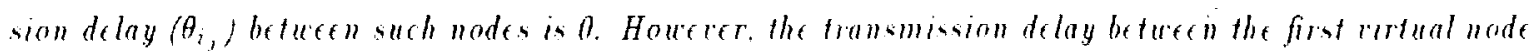
$\left(1, V_{i_{1}}\right.$ ) and its upstream neighbor (whech is also a cintual node) is $\theta_{i}$.

- If $x_{i}$ is an mactete node with no synchronous messages. it is transformed into a ritual node $\mathrm{Xl}_{\text {i }}$ as follous:

$$
T\left(X_{i}\right)=T^{*} X_{i}=\left(\left\{S_{d}\right\}, I_{i} \cdot \theta_{i}\right)
$$

where $S_{d}$ represents a stream of dummy syuchronous messages introduced into the cirtual node $1 \mathrm{r}$, with mesage length $C_{d}=0$ and periad $P_{d}=x$.

After transformation of the neturok. the cintual modes are connected in a ring fashrim.

Note that the total ring latency of the virtual network will be equal to that of the actual network from which it was derived. It is evident that the virtual network model is logically equivalent to the original network model.

\section{Appendix B Proofs of Protocol Timing Properties}

In this appendix, the proofs of Johnson and Sercik's theorem and the generalized Johnson and Sevcik's theorem will be presented. These theorems will be preceded by the definitions of a few terms and lemmas to be used in the their proofs.

\section{B.1 Definitions of Terms}

- $t_{i}(l) .(l=1.2 \ldots)$. It is the time when the coken makes its $l^{\text {th }}$ visit to node $i$.

- $R_{i}(l) .(l=1,2 \ldots)$. It is defined as follows:

$$
R,(l)= \begin{cases}t_{i}(l)+T T R T . & \text { if the token is early on its } l^{\text {h }} \text { visit to node } i: \\ R_{i}(l-1)+T T R T . & \text { otherwise. }\end{cases}
$$

That is $R,(l)$ indicates the next expected arrival time of the token at node $i$ after the token's $I^{\text {th }}$ visit. If the token is late on its $(l+1)^{\text {th }}$ vist to node $i$. then $R_{i}(l)$ will le the time at which $T R T_{i}$ : xpires and is reset to TTRT. Sote that the lefintions of $R_{i}(l)$ and $t_{i}(l)$ imply that

$$
R_{i}(l)-l_{i}(l) \leq T T R T
$$

- The amome of time left. hefore the intriation of the ring pecorery process by node $i$, can be expressed

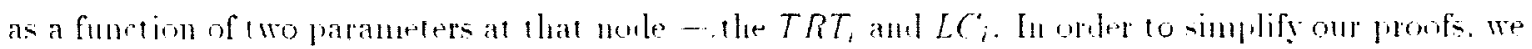

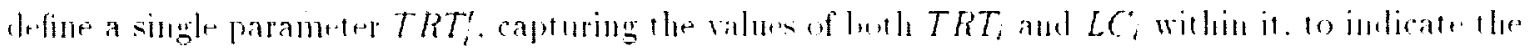

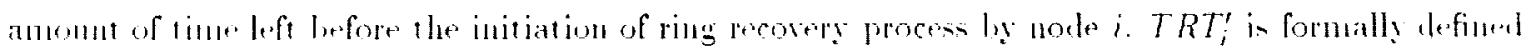
it) follow:

$$
T R T_{i}^{\prime}=T R T_{i}+\left(1-L C_{i}^{\prime}\right) \cdot T T R T \text {. }
$$


Given the fact that $0 \leq T R T_{i} \leq T T R T$ and $\theta \leq L C_{i} \leq 1$, it is clear that

$$
0 \leq T R T_{i}^{\prime} \leq 2 \cdot T T R T
$$

The physical meaning of $T R T_{i}^{\prime}$ is that when $T R T_{i}^{\prime}>T T R T$. the $T R T_{i}$ has not expired since the last token arrival. A token arring at this instant would le early. When $T R T_{i}^{\prime} \leq T T R T . L C_{i}^{\prime}=1$. Hence. the TRT, has expired once since the last token arrival. In either case, the amount of time left before node $i$ initiates the ring recovery process is $T R T_{i}^{\prime}$. In the event that $T R T_{i}^{\prime}$ becomes zero, the ring recovery process will be initiated.

\section{B.1 Proofs of Theorems 5.1 and 5.2 and Corollary 5.1}

In the proofs of the lemmas and theorems that follow. $T R T_{i}(t) . T H T_{i}(t) . L C_{i}(t) . T R T_{i}^{\prime}(t)$ represent the values of $T R T_{i}, T H T_{i}, L C_{i}$ and $T R T_{i}^{\prime}$ at time $t_{\text {. }}$

LEMMA B.1 For any integers $l>0 . r>0$ and any node $i(1 \leq i \leq n)$.

$$
R_{i}(l+\imath)-R_{i}(l) \leq \imath \cdot T T R T .
$$

The equality holds if the token arrival is late on its $l^{\text {th }} \cdot(l+1)^{\text {th }} \ldots$ and $(l+v-1)^{\text {th }}$ risits to node $i$.

The lemma can be easily proved by an induction argument.

LEMMA B.2 (Johnson's Lemma ['6]) After ring initialization. the TRT' ralues of all operational stations. will be monotonically increasing in the docenlink derection. up to and including the station uhich last reccired the tolen. ${ }^{5}$

The reader is referred to [26] for the proof of the ahove lemma.

Let us consider an example to illustrate the implication of the above lemma. Figure 3 blows the token is learing node $A$ and is enroute to node $B$ at some time $t$. Bi Johnson's Lemma the values of $T R T^{\prime}$ s are monotonically increasing in the downlink direction upto and including the station which last received the token (i.e. nole f). Threrefore.

$$
T R T_{B}^{\prime}<T R T_{C}^{\prime}<T R T_{D}^{\prime}<T R T_{E}^{\prime}<\ldots<T R T_{A}^{\prime}
$$

Sow, if the $T R T_{E}$ expires at this moment (i.e.. $T R T_{E}^{\prime} \leq T T R T$ ). (hen the TRTs of nodes B. C. $D$ will have also expired. Conseguently the coken will be late when it visits nodes B.C.D and $E$ for the first tinte after time $t$.

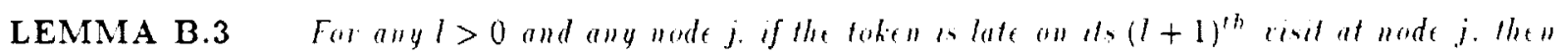

$$
l_{j}(l+l) \leq R_{j}(l)+T T R T-H_{j}
$$

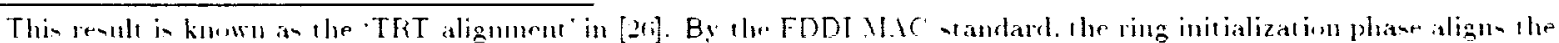

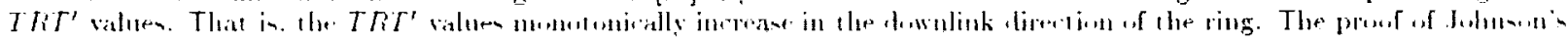

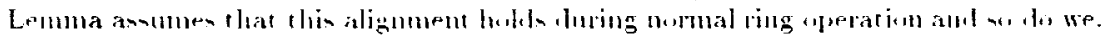


Proof: Let us first define the pluase wokeln is at node 7 " 10 mean that the token is heing held by node $i$ or is on its way to node $i$ from its upstream neightor (node $i-1$ if $i>1$, else node $n$ if $i=1$ ).

Recall that $T R T_{i}^{\prime}(t)$ is defined as

$$
T R T_{i}^{\prime}(t)=T R T_{i}(t)+\left(1-L C_{i}(t)\right) \cdot T T R T .
$$

If the token is late on its $(l+1)^{\text {th }}$ visit to node $j$. the token must be at some node $i$ at time $R_{j}(l)$. Assunte that the token arrives at node $i$ at time $T\left(T \leq R_{j}(l)\right)$. We have two cases to ronsider.

Case 1: The token arrives late at node $i$. In this case node $i$ will only transmit its syncluronous messages for at most $H_{i}$ time. Hence the token will leave node $i$ no later than $T+H_{i}+\theta_{i} \leq R_{j}(l)+H_{i}+\theta_{j}$.

Case 2: The token arrives early at node $i$. Therefore, $L C_{i}(T)=L C_{j}(T)=0$. By Johnson : Lemma (Lemma B.2) we have

$$
T R T_{i}^{\prime}(T)-T T R T \leq T R T_{j}^{\prime}(T)-T T R T
$$

From (51). we get

$$
T R T_{i}(T) \leq T R T_{j}(T)
$$

Thus.

$$
T H T_{i}(T)=T R T_{i}(T) \leq T R T_{j}(T)=R_{j}(l)-T
$$

Hence. node $i$ can transmit asynchronous messages for at most $R_{j}(l)-T$ time and transmit symchronous messages for at most $H_{i}$ time. In this case too. the token will leave node $i$ before

$$
T+\left(R_{j}(l)-T+H_{i}\right)+\theta_{i}=R_{j}(l)+H_{i}+\theta_{i}
$$

That is if the token is at node $i$ when $T R T_{j}$ expires. then the token will leave node $i$ no later than $R_{j}(l)+H_{i}+\theta_{i}$.

Nor consider the nodes on the way from node $i$ to node $j$. Let them be labeled as $n_{1} . n_{2} \ldots \ldots n_{k}$. According to Johnson s Lemma. the token will he late on its risit to each of these $k$ nodes on the way to node $j$. Hence. these nodes will transmit their sxuchronous messages only. That is the token will arrive at node $j$ no later than

$$
\begin{aligned}
R_{j}(l)+H_{j}+\theta_{i}+\sum_{h=1}^{k}\left(H_{n_{h}}+\theta_{n_{h}}\right)+\theta_{j}+\Delta & \leq R_{j}(l)+\sum_{h \neq j} H_{h}+\sum_{h=1}^{n} \theta_{h}+\Delta \\
& =R_{j}(l)+\sum_{h \neq j} H_{h}+\tau
\end{aligned}
$$

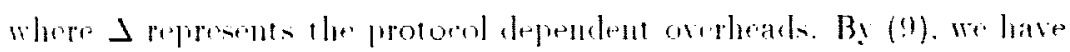

$$
t_{j}(l+1) \leq R_{j}(l)+T T R T-H_{\jmath}
$$

Q.E.D. 


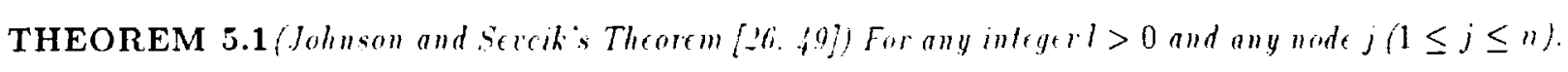

$$
t_{j}(l+1)-t_{j}(l) \leq 2 \cdot \operatorname{TTRT}-H_{j}
$$

Proof: If the token is not late at its $(l+1)^{\text {th }}$ visit to node $j \cdot$ then

$$
t_{j}(l+1)-t_{j}(l) \leq T T R T \leq 2 \cdot T T R T-H_{j}
$$

Otherwise. from Lemma B.3 we have

$$
\begin{aligned}
t_{j}(l+1)-t_{j}(l) & \leq R_{j}(l)+T T R T-H_{j}-t_{j}(l) \\
& \leq T T R T-H_{j}+\left(R_{j}(l)-t_{j}(l)\right) \\
& \leq 2 \cdot T T R T-H_{j}
\end{aligned}
$$

This simply says that the maximum time that can elapse between two consecutive token arrivals at some node is bounded by 2.TTRT-H. This result was first proved in [2(j]. Q.E.D.

THEOREM 5.2 (Generalied Johnson and Seciks Theorem) For any integerl $>0 . r>1$ and any node $j$ $(1 \leq j \leq n)$.

$$
t_{j}(l+i-1)-t_{j}(l) \leq i \cdot T T R T-H_{j}
$$

Proof: We prove the theorem by induction on $r$. For $r=2$. by Theorem 5.1 we have

$$
t_{j}(l+1)-t_{j}(l) \leq 2 \cdot T T R T-H_{j}
$$

Hence. the theorem holds for $v=2$.

Assume that for $t=h \cdot(66)$ holds, i.e..

$$
t_{j}(l+k-1)-t_{j}(l) \leq k \cdot T T R T-H_{j}
$$

Now we consider for $c=k+1$. We have two cases:

Case 1: The token arrives early on its $\mathbf{v}^{\prime}$ th visit to node $\mathbf{j}\left(\mathbf{l}<\mathbf{v}^{\prime}<\mathbf{v}\right)$. That is.

$$
R_{j}\left(\iota^{\prime}-1\right) \geq f_{j}\left(r^{\prime}\right)
$$

Hence.

$$
\begin{aligned}
t_{j}\left(c^{\prime}\right)-t_{j}(l) & =\left(t_{j}\left(\iota^{\prime}\right)-R_{j}(l)\right)+\left(R_{j}(l)-t_{j}(l)\right) \\
& \leq\left(R_{j}\left(c^{\prime}-1\right)-R_{j}(l)\right)+\left(R_{j}(l)-t_{j}(l)\right)
\end{aligned}
$$

By Lemma B.I and (50), we lave

$$
l_{j}\left(r^{\prime}\right)-l_{j}(l) \leq\left(c^{\prime}-1-l\right) \cdot T T R T+T T R T=\left(r^{\prime}-l\right) \cdot T T R T .
$$

Sow

$$
t_{j}(l+(k+1)-1)-t_{j}\left(c^{\prime}\right)=t_{j}\left(c^{\prime}+\left(l-r^{\prime}+k+1\right)-1\right)-t_{j}\left(c^{\prime}\right) .
$$


Bỵ the induction hypothesis $(68)$ and $(72)$, we have

$$
t_{j}(l+(k+1)-1)-t_{j}\left(\imath^{\prime}\right) \leq\left(l-v^{\prime}+k+1\right) \cdot T T R T-H_{j} .
$$

Adding (7I) and (73) on both sides, we have

$$
\begin{aligned}
t_{j}(l+(k+1)-1)-t_{j}(l) & \leq\left(l^{\prime}-l\right) \cdot \operatorname{TTRT}-H_{j}+\left(l-v^{\prime}+k+1\right) \cdot \operatorname{TTRT} \\
& =(k+1) \cdot \operatorname{TTRT}-H_{j}
\end{aligned}
$$

Thus, the theorem is proved.

Case 2: The token is always late at node $j$ between the $(l+1)^{\text {th }}$ visit and the $(l+k)^{\text {th }}$ visit inclusive.

Because the token is late, from Lemma B.3. we have

$$
t_{j}(l+k)=t_{j}(l+(k+1)-1) \leq R_{j}(l+k-1)+T T R T-H_{j} .
$$

Therefore. by (.50) and (5.5).

$$
\begin{aligned}
t_{j}(l+(k+1)-1)-t_{j}(l) & =\left(t_{j}(l+(k+1)-1)-R_{j}(l)\right)+\left(R_{j}(l)-t_{j}(l)\right) \\
& \leq\left(R_{j}(l+k-1)+T T R T-H_{j}-R_{j}(l)\right)+T T R T \\
& =R_{j}(l+k-1)-R_{j}(l)+2 \cdot T T R T-H_{j} \\
& =(k-1) \cdot T T R T+2 \cdot T T R T-H_{j} \quad(b j \mathrm{~L} \\
& =(k+1) \cdot T T R T-H_{j} .
\end{aligned}
$$

This concludes the proof of the theorem.

COROLLARY 5.1 . Assume that at time t, a synchronous mesage with perod $P_{i}$ arroces at node $i(1 \leq$ $i \leq n)$. Then. in the time interral $\left(t . t+P_{i}\right)$ the total amount of time $\left(\mathrm{X}_{i}\right)$ aralable for node i to tranamet this synchronous measage is bounded by

$$
x_{i} \geq\left\lfloor\frac{P_{i}}{T T R T}-1\right\rfloor \cdot H_{i}
$$

In the worst cane. the louter bound will be tight if

$$
\varepsilon_{i} \geq H_{i}
$$

where $\dot{c}_{i}=\left\lceil\frac{P_{1}}{T T R T}\right\rceil \cdot T T R T-P_{i}$

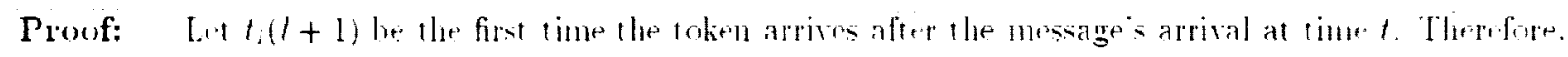

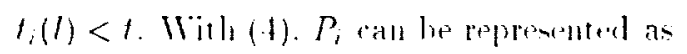

$$
P_{i}=m_{i} \cdot T T R T-r_{i}
$$

where $m_{i}=\left\lceil\frac{P_{2}}{T T K T}\right\rceil$ and $0 \leq \delta_{i}<T T R T$. Wi haw two and to comider: 
Case 1: $0<\delta_{1}<T T R T$ : This implips

$$
\begin{aligned}
P_{i} & >\left(m_{i}-1\right) \cdot T T R T & & \\
& =\left(m_{i}-1\right) \cdot T T R T-H_{i}+H_{i} & & \\
& \geq t_{i}\left(l+m_{i}-2\right)-t_{i}(l)+H_{i} & & \text { (hy Theorem } 5.2) \\
& \geq t_{i}\left(l+m_{i}-2\right)-t+H_{i} . & & \text { (since } \left.t_{i}(l)<t\right)
\end{aligned}
$$

This means

$$
1+P_{i} \geq t_{i}\left(l+m_{i}-2\right)+H_{i}
$$

Hence. by the end of the message's period (i.e., $t+P_{i}$ ) the token will have made $m_{i}-2$ visits to note $i$ since time $t$. In each of these visits, node $i$ can transmit its sychchonous message for the allocated synchronous capacity $H_{i}$. Consequently. the total amount of the time for node $i$ to transmit this sunchronons message will lie at least"

$$
\left.\left(m_{i}-2\right) \cdot H_{i}=\left\lfloor\frac{P_{i}}{T T R T}-1\right\rfloor \cdot H_{i} . \quad \text { (since }\left(m_{i}-1\right) \cdot T T R T<P_{i}<m_{i} \cdot T T R T\right)
$$

Case 2: $\delta_{1}=0$ : This implies that

$$
\begin{aligned}
P_{i} & =m_{i} \cdot T T R T & & \\
& =m_{i} \cdot T T R T-H_{i}+H_{i} & & \\
& \geq t_{i}\left(l+m_{i}-1\right)-t_{i}(l)+H_{i} & & \text { (by Theorem } 5.2) \\
& \geq t_{i}\left(l+m_{i}-1\right)-t+H_{i} . & & \text { (since } \left.t_{i}(l)<t\right)
\end{aligned}
$$

This means

$$
t+P_{i} \geq t_{i}\left(l+m_{i}-1\right)+H_{i}
$$

Hence. by the end of the message s period (i.e.. $t+P_{i}$ ) the token will have made $m_{i}-1$ risits to node $i$ since time $f$. In each of these visits node $i$ can transmit its synchronous message for the allocated synchronous capacity $H_{i}$. Consecuently. the total amount of the time for node $i$ to transmit this sinchronous message will be at least

$$
\begin{aligned}
\left(m_{i}-1\right) \cdot H_{i} & =\left\lceil\frac{P_{i}}{T T R T}-1\right\rceil \cdot H_{i} \\
& =\left\lfloor\frac{P_{i}}{T T R T}-1\right\rfloor \cdot H_{i} \quad \text { (since } \frac{P_{i}}{T T R T} \text { is an integral number). }
\end{aligned}
$$

From Cases 1 and 2. we see that (ii) holds.

Sow consider the case when (is) holds i.e.. $\delta_{i} \geq H_{i}$.

$$
c_{i}=m_{i} \cdot \operatorname{TTRT}-P_{i} \geq H_{i} .
$$

Recall that $t_{i}(l+1)$ is the firs time the tuken arrives after the message s arrival at time $t$. Let $t=t_{i}(l)+\epsilon$ $(\epsilon>0)$. This inplien in the mors case

$$
\begin{aligned}
& P_{i} \leq m_{i} \cdot \operatorname{TTRT}-H_{i} \\
& =t_{1}\left(l+m_{i}-1\right)-t_{i}(l) \\
& =t_{1}\left(t+m_{i}-1\right)-t+t \text {. }
\end{aligned}
$$

\footnotetext{
"Wute that since", $>U . m_{1} \geq 3$.
} 
Consequently.

$$
1+P_{i} \leq t_{i}\left(l+m_{i}-1\right)+\epsilon
$$

Because of the arhitrarity of $t$. we can let $t-t_{i}(l)^{+}$. That is. $t-0$. Therefore,

$$
t+P_{i} \leq t_{i}\left(l+m_{i}-1\right)
$$

The above inequality indicates that the $\left(l+m_{i}-1\right)^{\text {th }}$ visit of the token will not he earlier than $t+P_{i}$. IIence. in the time interval $\left(t . t+P_{i}\right)$. the node will have no more than $m_{i}-2\left(=\left\lfloor\frac{P_{1}}{T T R T}-1\right\rfloor\right)$ risits of the token. Thus the lower hound of $X_{i}$ is tight. Q.E.D.

\section{Appendix C Proofs of Lemma 6.1 and Theorem 6.3}

\section{C.1 Proof of Le'mma 6.1}

LEMMA 6.1 For any synchronous mesage stream $i(1 \leq i \leq n)$ we have

$$
\frac{\left\lfloor\frac{P_{1}}{T T R T}-1\right\rfloor}{P_{i} / T T R T} \geq \frac{1}{3-\frac{1}{T T R T}} \geq \frac{1}{3} .
$$

Proof: From (4), we have

$$
P_{i}=m_{i} \cdot T T R T-\hat{\kappa}_{i}
$$

where $m_{i}=\left\lceil\frac{P_{1}}{T T R T}\right\rceil$ and $\varepsilon_{i}=\left\lceil\frac{P_{i}}{T T R T}\right\rceil \cdot T T R T-P_{i}$. Depending on the value of $\varepsilon_{i}$. we have two cases to consider:

Case 1: $0<\dot{\varepsilon}_{i}<$ TTRT. This implies $m_{i} \geq 3$. We have

$$
\begin{aligned}
\frac{\left\lfloor P_{i} / T T R T-1\right\rfloor}{P_{i} / T T R T} & =\frac{\left\lfloor\frac{\left\lfloor m_{i} T T R T-i_{i}\right)}{T T h T}-1\right\rfloor}{\left(m_{i} \cdot T T R T-\dot{s}_{i}\right) / T T R T} \\
& =\frac{\left\lfloor m_{i}-\frac{s_{i}}{T T R T}-1\right\rfloor}{m_{i}-\frac{i}{T T R T}}=\frac{m_{i}-2}{m_{i}-\frac{s_{1}}{T T R T}} .
\end{aligned}
$$

Tote that the right hand side of $(92)$ is an increasing function of $m_{i}{ }^{i}$ Therefore. the minimum value of (92) is obtained hy substituting the minimum ralue of $m_{i}$. i.e... $m_{i}=3$. Hence

$$
\frac{\left\lfloor P_{i} / \text { TTRT }-1\right\rfloor}{P_{i} / \text { TTRT }}=\frac{m_{i}-2}{m_{i}-\frac{i}{T T K T}} \geq \frac{1}{3-\frac{i}{T T R T}} .
$$

Furtler. the right hand side of $(94)$ is an increasing function of $\dot{\varepsilon}_{i}$. If we let $\dot{\varepsilon}_{1}-0^{+}$. We have

$$
\frac{\left\lfloor P_{1} / \text { TTRT }-1\right\rfloor}{P_{1} / \text { TTRT }} \geq \frac{1}{3-\frac{s}{T T I T}} \geq \frac{1}{3} \text {. }
$$

Thun. her lemmat holk in hlis cast.

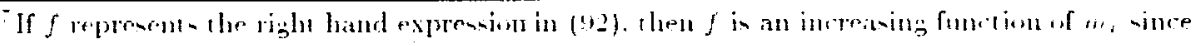

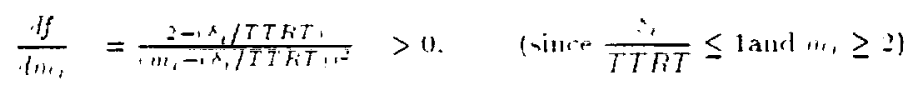


Case 2: $\delta_{2}=0$. This implies $P_{i}=m_{i} \cdot T T R T$. We have

$$
\frac{\left\lfloor P_{i} / T T R T-1\right\rfloor}{P_{i} / T T R T}=\frac{m_{i}-1}{m_{i}} \geq \frac{1}{2}
$$

From (9.j) and (96). we get

$$
\frac{\left\lfloor P_{1} / T T R T-1\right\rfloor}{P_{i} / T T R T} \geq \frac{1}{2} \geq \frac{1}{3-\frac{1}{T T R T}} \geq \frac{1}{3}
$$

Q.E.D.

\section{C.2 Proof of Theorem 6.3}

In this sulsection. a proof of theorem 6.3 is presented. We need to prove a lemma first.

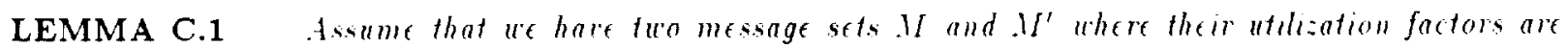
equal. i.f. $C(M)=C\left(M J^{\prime}\right)$. Further. assume that the synchromous capacity allocated to all the nodes is the same irrespectile of the message set consideved i.e. for $i=1 \ldots$ n.

$$
H_{i}^{\prime}=H_{i}
$$

The first message set is arbitrary. That is.

$$
M=\left\{\left(C_{1}, P_{1}\right) \ldots\left(C_{i}, P_{i}\right) \ldots\left(C_{n}, P_{n}\right)\right\} .
$$

By (4). a message period $P_{i}$ is of the form

$$
P_{i}=m_{i} \cdot T T R T-\varepsilon_{i}
$$

where $m_{i}=\left\lceil\frac{P_{i}}{T T R T}\right\rceil$ and $0 \leq \varepsilon_{i}<T T R T$.

The second mesage set. $Y^{\prime}$ is of the form

$$
U^{\prime}=\left\{\left(C_{1}^{\prime} \cdot P_{1}^{\prime}\right) \ldots\left(C_{i}^{\prime \prime} \cdot P_{i}^{\prime}\right) \ldots\left(C_{n}^{\prime \prime} \cdot P_{n}^{\prime}\right)\right\}
$$

The parameters of the mesages in . ${ }^{\prime} d e p e n d$ on those in .Y and $H_{\text {i }}$ as folloecs:

$$
P_{i}^{\prime}= \begin{cases}P_{i} . & \text { if } m_{i}=2 \\ m_{i} \cdot T T R T-H_{i} . & \text { if } m_{i} \geq 3\end{cases}
$$

and

$$
C_{i}^{\prime}= \begin{cases}C_{i}^{\prime} & \text { if } m_{i}=2 \\ P_{i}^{\prime} \cdot \frac{\ddot{P_{i}}}{} & \text { if } m_{i} \geq 3 .\end{cases}
$$

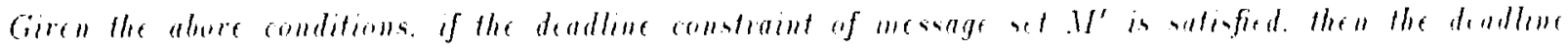

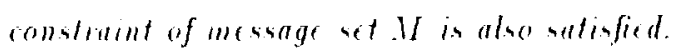

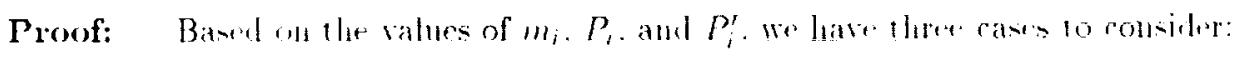

$$
\text { - } m_{i}=? \text {. }
$$


- $m_{i} \geq 3$ and $P_{i} \leq P_{i}^{\prime}$, and

- $m_{i} \geq 3$ and $P_{i}>P_{i}^{\prime}$.

In each of these three cases we show that if there is sufficient time to successfully transmit message $\left(C^{\prime}, P_{i}^{\prime}\right)$ in message set $W^{\prime}$. then the time arailable is also sufficient to transmit message $\left(C_{i}^{\prime} . P_{i}^{\prime}\right)$ in.$M$.

Case 1: $m_{t}=2$ By $(102)$ and (103). we have

$$
P_{i}^{\prime}=P_{i} \cdot C_{i}^{\prime}=C_{i} . \text { and } H_{i}^{\prime}=H_{i} .
$$

Therefore, the lower bound on the time available to transmit hoth $\left(C_{i}^{\prime}, P_{i}\right)$ and $\left(C_{,}^{\prime \prime} . P_{i}^{\prime}\right)$ will he the same. Since the deadline constraint is not riolated when transmitting any message in message set .$I^{\prime}$. the deadline constraint will not be violated when transmitting a message $\left(C_{i} . P_{i}\right)\left(=\left(C_{i}^{\prime \prime} . P_{i}^{\prime}\right)\right)$ in message set $M$ either.

Case 2: $m_{1} \geq 3$ and $P_{1} \leq P_{1}^{\prime}$. By (102). we have

$$
\delta_{i} \geq \delta_{i}^{\prime}=H_{i}^{\prime}>0 \text {. }
$$

From (10:3). we have

$$
C_{i}^{\prime}=P_{i}^{\prime} \cdot \frac{C_{i}^{\prime}}{P_{i}}=C_{i} \cdot \frac{P_{i}^{\prime}}{P_{i}} \geq C_{i} .
$$

Ising Corollary 5.1, we claim that the lower bound on the time available to transmit either message $\left(C_{i}, P_{i}\right)$ or $\left(C_{i}^{\prime}, P_{i}^{\prime}\right)$ during their respective message periods $P_{i}$ and $P_{i}^{\prime}$ is the same. This is because $X_{i}=X_{i}^{\prime}=\left(m_{i}-2\right) \cdot H_{i}=\left(m_{i}-2\right) \cdot H_{i}^{\prime}$. Since this amount of time is sufficient to transmit a message of length $C_{i}^{\prime}$, the message with length $C_{i}$ can also be transmitted before the end of period $P_{i}$. That is. the deadline constraint of messages in this case is met.

Case 3: $m_{1} \geq 3$ and $P_{1}>P_{1}^{\prime}$. Let

$$
P_{i}=P_{i}^{\prime}+\theta \quad\left(0<\theta<H_{i}^{\prime}\right) .
$$

From (103) and $(10 \bar{t})$. we have

$$
C_{i}=C_{i}^{\prime}\left(1+\frac{\theta}{P_{i}^{\prime}}\right)
$$

Yow as seen in the proof of Corollary 5.1 the $(m-1)^{\text {th }}$ token arrival at noule $i$, in the worst case ocents at the end of the period $P_{i}^{\prime}=m_{i} \cdot T T R T-H_{i}$. Hence. When $P_{i}=P_{i}^{\prime}+\theta$. the nod $i$ ran ransmit additional sunchronous nessages for a time $\theta$. That is the maximum anount (say $X_{2}$ ) of time available to transmit symchronous messages within period $P_{i}$. in the wort case is $C_{i}^{\prime}+\theta$. Therefore.

$$
\begin{aligned}
& x_{i}=C_{i}^{\prime}+\theta \geq C_{i}^{\prime}+\frac{C_{i}^{\prime}}{P_{i}^{\prime}} \theta \quad\left(\text { ine } \frac{C_{i}^{\prime}}{P_{i}^{\prime}} \leq 1\right) \\
& =\left(\because\left(1+\frac{\theta}{P_{i}^{\prime}}\right)\right. \\
& =C_{i} \text {. }
\end{aligned}
$$

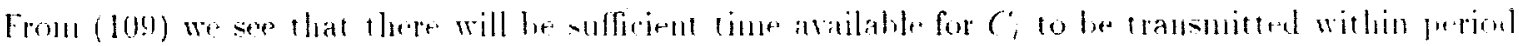
$P_{i}$. That is the deatline constraint of the mesage set. M is sat isfied in this case too. 
From the results of the ahove cases, we see that the dealline constraint of message set If is satisfied if the deadline constraint of message set . $W^{\prime}$ is satisfied. Q.E.D.

We are now ready to prove Theorem 6.3 .

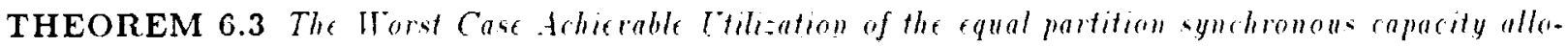
cation scheme is $\frac{1}{3 n-(1-a)} \cdot(1-a)$ where $a=\frac{T}{\text { TThT }}$ and $n$ is the number of nodes.

Proof: We prove the theorem by showing that the following statements are true:

1. For any message set.$M$, the protocol constraint will always be satisfied.

2. For any message set $M$ with utilization factor $l(. M) \leq \frac{1}{3 n-(1-a)} \cdot(1-a)$. the deaclline constraint will be satisfied.

3. For any given $\epsilon>0$, there exists a message set. II with utilization factor $C^{-}(M)=\frac{1}{3 n-(1-a)}(1-n)+\epsilon$. so that the deadline constraint cannot be satisfied for this set of messages when the synchronous capacities are allocated by using the equal partition scheme.

Proof of Statement 1: From (26). we have

$$
\sum_{i=1}^{n} H_{i}=n \cdot \frac{T T R T-T}{n}=T T R T-\tau .
$$

That is the protocol constraint is always satisfied.

Proof of Statement 2: Consider a message set $M=\left\{\left(C_{1}, P_{1}\right) \ldots\left(C_{i}, P_{i}\right) \ldots\left(C_{n}, P_{n}\right)\right\}$ with utilization factor

$$
\left[\leq \frac{1}{3 n-(1-a)} \cdot(1-a)\right.
$$

whele $n$ is the number of nodes and $a=\frac{T}{T T R T}$. Further. any period $P$ in message set $I I$ can be expresied in the form given by (4). That is.

$$
P_{i}=m_{i} \cdot T T R T-\varepsilon_{i}
$$

where $m_{i}=\left\lceil\frac{P}{T T R T}\right\rceil \geq 2$ and $0 \leq \varepsilon_{i}<T T R T$. Now construct a message set $. W^{\prime}=\left\{\left(C_{1}^{\prime}, P_{1}^{\prime}\right) \ldots\left(C_{i}^{\prime}, P_{i}^{\prime}\right) \ldots\left(C_{n}^{\prime}, P_{n}^{\prime}\right)\right\}$ where

$$
P_{i}^{\prime}= \begin{cases}P_{i} . & \text { if } m_{i}=2: \\ m_{i} \cdot T T R T-H_{i}^{\prime} . & \text { if } m_{i} \geq 3\end{cases}
$$

and

$$
r_{i}^{\prime}= \begin{cases}C_{i} & \text { if } m_{i}=2 . \\ P_{i}^{\prime} \cdot \frac{\dot{P}_{i}}{p_{i}} & \text { if } m_{i} \geq 3 .\end{cases}
$$

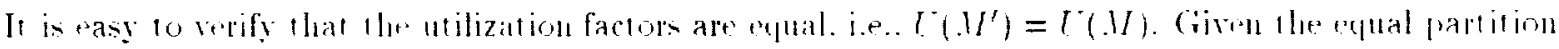

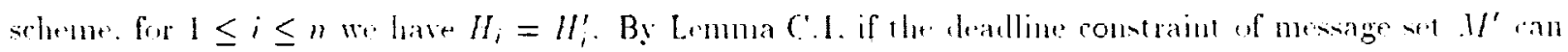

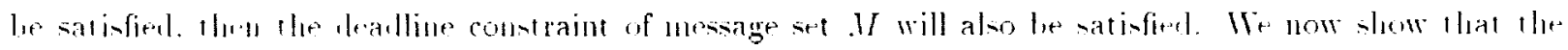
dealline comstraint of message set $I^{\prime}$ is ativfied. 
Case 1: We first consider the messages whose periods are given by

$$
P_{i}^{\prime}=m_{i} \cdot T T R T-H_{i}^{\prime}
$$

where $m, \geq 3$.

Multiplying hoth sides of (111) ly $C^{\prime} / /$, we have

$$
\begin{aligned}
C_{i}^{\prime} & \leq \frac{1}{3 n-(1-a)} \cdot \frac{C_{i}^{\prime}}{C^{\prime}} \cdot(1-a) . \\
& \leq \frac{1}{3 n-(1-a)} \cdot \frac{C_{i}^{\prime}}{C_{i}^{\prime \prime} / P_{i}^{\prime}} \cdot(1-a) \\
& =\frac{1}{3 n-(1-a)} \cdot P_{i}^{\prime} \cdot(1-a)=\frac{1}{3-\frac{1-a}{n}} \cdot \frac{1}{n} \cdot P_{i}^{\prime} \cdot(1-a) .
\end{aligned}
$$

Substituting $\frac{1-a}{n}$ with $\frac{H_{i}}{T T R T}$ (since $H_{i}^{\prime}=H_{i}=\frac{T T R T ! 1-a)}{n}$ ). We obtain

$$
C_{i}^{\prime} \leq \frac{1}{3-\frac{H_{i}}{T T R T}} \cdot \frac{1}{n} \cdot P_{i}^{\prime} \cdot(1-a)
$$

From Lemma 6.1 and (113) we have

$$
\frac{1}{3-\frac{H}{T T R T}} \leq \frac{\left\lfloor P_{i}^{\prime} / T T R T-1\right\rfloor}{P_{i}^{\prime} / T T R T}
$$

Therefore, we can rewrite (117) as

$$
\begin{aligned}
C_{i}^{\prime} & \leq \frac{\left\lfloor P_{i}^{\prime} / T T R T-1\right\rfloor}{P_{i}^{\prime} / T T R T} \cdot \frac{1}{n} \cdot P_{i}^{\prime} \cdot(1-a) \\
& =\left\lfloor\frac{P_{i}^{\prime}}{T T R T}-1\right\rfloor \cdot \frac{T T R T}{n} \cdot(1-a) \\
& =\left\lfloor\frac{P_{i}^{\prime}}{T T R T}-1\right\rfloor \cdot H_{i} .
\end{aligned}
$$

Because of Corollary j.1. inequality (119) shows that the deadline constraint of the messages in this case is always satisfied.

Case 2: We now ronsider those messages whose periods are given by

$$
P_{i}^{\prime}=P_{i}=\ddot{2} \cdot T T R T \text {. }
$$

By (111). we have

$$
l \leq \frac{1-n}{3 n-(1-a)} \leq \frac{1-n}{2 n}
$$

wher $n \geq 1$

Multiplying boh siden of $(121)$ by $\frac{C^{\prime \prime}}{P^{\prime} l^{\prime}}$, We have

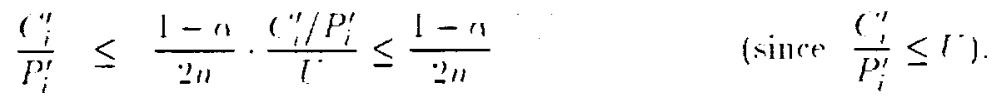


Thus

$$
\begin{aligned}
C_{i}^{\prime} & \leq P_{i}^{\prime} \cdot \frac{1-a}{2 n} \\
& =\frac{T T R T \cdot(1-a)}{n} \\
& =H_{i}^{\prime} \\
& =\left\lfloor\frac{2 \cdot T T R T}{T T R T}-1\right\rfloor \cdot H_{i}^{\prime} \\
& =\left\lfloor\frac{P_{i}^{\prime}}{T T R T}-1\right\rfloor \cdot H_{i}^{\prime} .
\end{aligned}
$$

Because of Corollary j.1, (123) implies that the deadline constraint of message set $W^{\prime}$ is satiffied.

From Cases 1 and 2. we see that the deadline constraint of message set $M^{\prime}$ is satisfied when $C^{\prime}\left(. Y^{\prime}\right) \leq$ $\frac{1-a}{3 n-(1-a)}$. By Lemma C.I. the deadline constraint of message set . I is also satisfied.

Proof of statement 3: For any given $\epsilon>0$. there exists a message set $M$ with utilization factor $Y(M)=$ $\frac{1}{3 n-(1-a)}(1-a)+\epsilon$ so that the deadline constraint cannot be satisfied for this set of messages when the synchronous capacities are allocated using the equal partition scheme.

Let $\operatorname{TTRT}=P_{\min } / 2=1 / 2$ and $n$ be the number of nodes. For any given $\epsilon>0$. consider the following message set:

$$
\begin{aligned}
& C_{1}=\epsilon / 3 . \quad P_{1}=1 . \\
& C_{2}=\frac{1-a}{2 n}+\frac{(\epsilon / 3) \mid 3 n-(1-n))}{2 n} . \quad P_{2}=\frac{3}{2}-\frac{1-n}{2 n} . \\
& C_{i}=c / 3 . \quad P_{i}=(n-2) . \quad \text { for } \mathrm{i}=3 \ldots \mathrm{n} \text {. }
\end{aligned}
$$

The total utilization factor of the above message set is

$$
\begin{aligned}
I^{-} & =\sum_{i=1}^{n} \frac{C_{i}}{P_{i}}=\left(\frac{\epsilon}{3}\right)+\left(\frac{\frac{1-n}{2 n}+\frac{(\epsilon / 3) 3 n-11-n n)}{2 n}}{\frac{3}{2}-\frac{1-n}{2 n}}\right)+\left(\sum_{i=3}^{n} \frac{\epsilon / 3}{n-2}\right) \\
& =\left(\frac{\epsilon}{3}\right)+\left(\frac{1-n}{3 n-(1-n)}+\frac{\epsilon}{3}\right)+\left(\frac{\epsilon}{3}\right) \\
& =\frac{1-n}{3 n-(1-n)}+\epsilon .
\end{aligned}
$$

We now show that $\varepsilon_{2} \geq H_{2}$. We have.

$$
\begin{aligned}
\delta_{2}=\left\lceil\frac{P_{1}}{T T R T}\right\rceil \cdot T T R T-P_{2} & =\left\lceil\frac{3 / 2-(1-n) / 2 n}{1 / 2}\right\rceil \cdot \frac{1}{2}-\left(\frac{3}{2}-\frac{1-n}{2 n}\right) \\
& =\lceil 3-(1-n) / n\rceil \frac{1}{2}-\frac{3}{2}+\frac{1-n}{2 n} \\
& =\frac{3}{2}-\frac{3}{2}+\frac{1-n}{\frac{2 n}{T}} \\
& =\frac{1-a}{2 n}=\frac{T T R T \cdot(1-n)}{n} \geq H_{2} .
\end{aligned}
$$

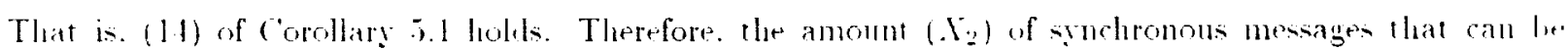

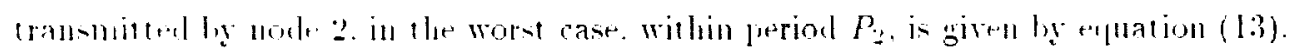

$$
x_{2}=\left\lfloor\frac{P_{2}}{T T R T}-1\right\rfloor \cdot H_{2}
$$




$$
\begin{aligned}
& =\left\lfloor\frac{(3 / 2)-((1-a) / 2 n)}{1 / 2}-1\right\rfloor \cdot \frac{1-a}{2 n} \\
& =\left\lfloor 3-\frac{1-a}{n}-1\right\rfloor \cdot \frac{1-a}{2 n} \\
& =\frac{1-n}{2 n}<C_{2} .
\end{aligned}
$$

We see that the deadline constraint has been violated when the utilization factor of the message set is greater than $\frac{1-a}{3 n-(1-a)}$ Q.E.D. 


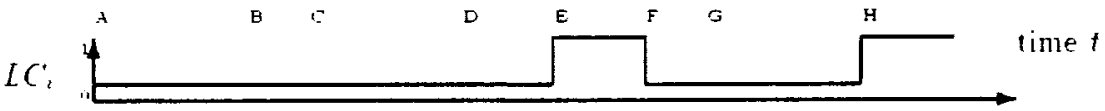

A: $T R T_{1}$ is resel 10 TTRT. $L C_{1}-0$. Token is released.

B: Token is recieved early. THT $-T R T_{1}$. TRT, - TTRT. IC; -0.

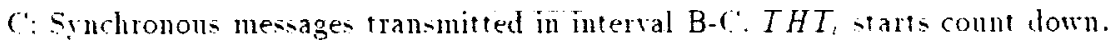

D: Asyuchronous messages transmitted in interval $(-D$. Token released.

E: TRT, expires and is reset to TTRT $L C,-1$.

F: Token received late since $L C_{2}^{\prime}=1 . L C_{1}-0$. Transmit sunchronous frames only.

G: Allocated capacity for stnchronons tran-mision expires. Token is released.

H: $T R T$, expires again and is rent to TTRT, $L C^{\prime},-1$.

Finure 1: lu Example of TRT, and Le': Nersu-Timet 


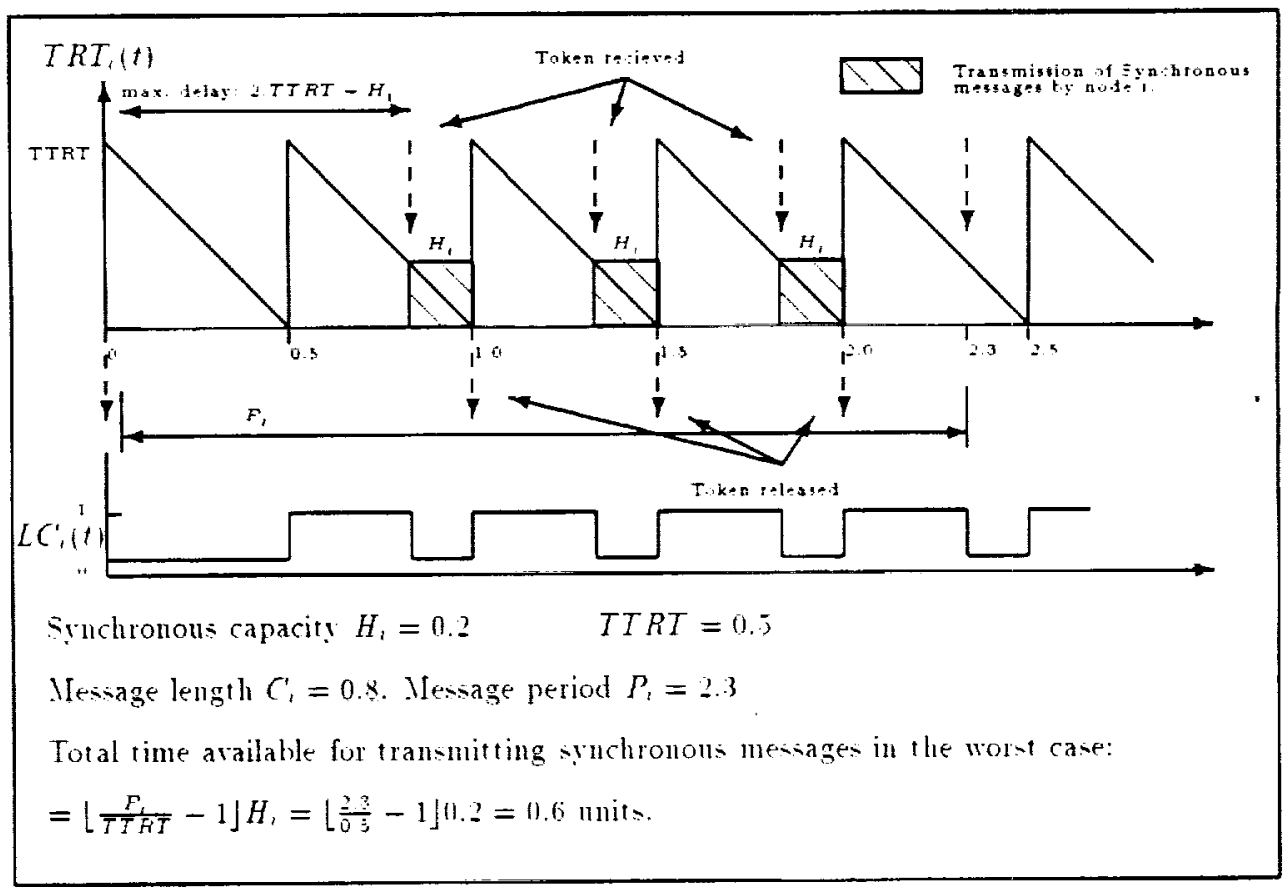

Finure 2: Tuken Arrivalo at Vole $i$ in a Wowt Case Situation 


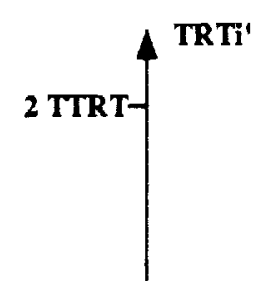

$-$

$$
-
$$$$
-
$$$$
-
$$$$
\text { - }
$$$$
\text { - }
$$

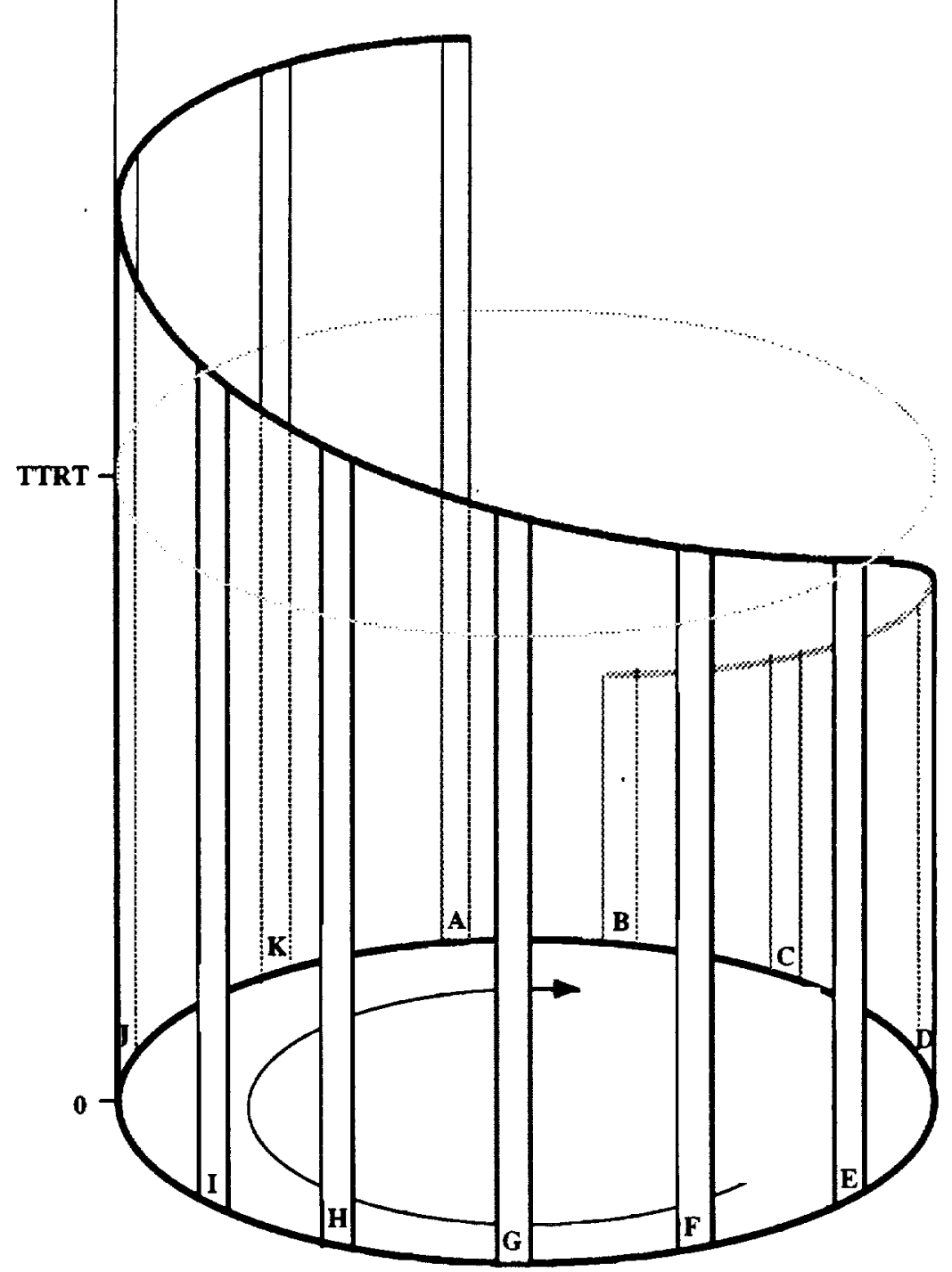

Figure 3: An Illus ration of TRT . Ilignmen 
$\bar{E}$

$\bar{\equiv}$

-

$\bar{\sigma}$

$\overline{\underline{\underline{\underline{\underline{\underline{a}}}}}}$

$\bar{E}$

$\underline{\underline{\underline{\underline{\underline{\underline{e}}}}}}$

$\bar{E}$

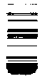

$\underline{\underline{\underline{\underline{\underline{\underline{E}}}}}}$

言

ए

$\underline{\overline{\underline{\underline{\underline{E}}}}}$

衰

$\equiv$

$\underline{\bar{E}}$

$\underline{\underline{\underline{\underline{\underline{2}}}}}$

严

$=$

$\varnothing$ 